\title{
Structural Characterization of CYP51 from Trypanosoma cruzi and Trypanosoma brucei Bound to the Antifungal Drugs Posaconazole and Fluconazole
}

\author{
Chiung-Kuang Chen ${ }^{1}$, Siegfried S. F. Leung ${ }^{1}$, Christophe Guilbert ${ }^{1}$, Matthew P. Jacobson ${ }^{1}$, James H. \\ McKerrow $^{2}$, Larissa M. Podust ${ }^{1 *}$
}

1 Department of Pharmaceutical Chemistry, University of California, San Francisco, California, United States of America, 2 Sandler Center for Basic Research in Parasitic Diseases, University of California, San Francisco, California, United States of America

\begin{abstract}
Background: Chagas Disease is the leading cause of heart failure in Latin America. Current drug therapy is limited by issues of both efficacy and severe side effects. Trypansoma cruzi, the protozoan agent of Chagas Disease, is closely related to two other major global pathogens, Leishmania spp., responsible for leishmaniasis, and Trypansoma brucei, the causative agent of African Sleeping Sickness. Both T. cruzi and Leishmania parasites have an essential requirement for ergosterol, and are thus vulnerable to inhibitors of sterol $14 \alpha$-demethylase (CYP51), which catalyzes the conversion of lanosterol to ergosterol. Clinically employed anti-fungal azoles inhibit ergosterol biosynthesis in fungi, and specific azoles are also effective against both Trypanosoma and Leishmania parasites. However, modification of azoles to enhance efficacy and circumvent potential drug resistance has been problematic for both parasitic and fungal infections due to the lack of structural insights into drug binding.

Methodology/Principal Findings: We have determined the crystal structures for CYP51 from T. cruzi (resolutions of $2.35 \AA$ and $2.27 \AA$ ), and from the related pathogen T. brucei (resolutions of $2.7 \AA$ and $2.6 \AA$ ), co-crystallized with the antifungal drugs fluconazole and posaconazole. Remarkably, both drugs adopt multiple conformations when binding the target. The fluconazole 2,4-difluorophenyl ring flips $180^{\circ}$ depending on the $\mathrm{H}$-bonding interactions with the BC-loop. The terminus of the long functional tail group of posaconazole is bound loosely in the mouth of the hydrophobic substrate binding tunnel, suggesting that the major contribution of the tail to drug efficacy is for pharmacokinetics rather than in interactions with the target.
\end{abstract}

Conclusions/Significance: The structures provide new insights into binding of azoles to CYP51 and mechanisms of potential drug resistance. Our studies define in structural detail the CYP51 therapeutic target in T. cruzi, and offer a starting point for rationally designed anti-Chagasic drugs with improved efficacy and reduced toxicity.

Citation: Chen C-K, Leung SSF, Guilbert C, Jacobson MP, McKerrow JH, et al. (2010) Structural Characterization of CYP51 from Trypanosoma cruzi and Trypanosoma brucei Bound to the Antifungal Drugs Posaconazole and Fluconazole. PLoS Negl Trop Dis 4(4): e651. doi:10.1371/journal.pntd.0000651

Editor: Timothy G. Geary, McGill University, Canada

Received November 11, 2009; Accepted February 16, 2010; Published April 6, 2010

Copyright: (c) 2010 Chen et al. This is an open-access article distributed under the terms of the Creative Commons Attribution License, which permits unrestricted use, distribution, and reproduction in any medium, provided the original author and source are credited.

Funding: This work was supported by the NIH RO1 grants GM078553 (to LMP) and GM086602 (to MPJ), NIAD-TDRU (Tropical Disease Research Unit) Al35707 (to JMK) and The Sandler Family Foundation. CG was supported by the NIH RO1 grant Al46967 to Prof. Thomas L. James. The Advanced Light Source is supported by the Director, Office of Science, Office of Basic Energy Sciences, of the U.S. Department of Energy under Contract No. DE-AC02-05CH11231. The funders had no role in study design, data collection and analysis, decision to publish, or preparation of the manuscript.

Competing Interests: Matthew P. Jacobson is a consultant to Schrodinger, LLC.

*E-mail: larissa.podust@ucsf.edu

\section{Introduction}

Chagas Disease, a potentially lethal tropical infection, is caused by the kinetoplastid protozoan Trypanosoma cruzi, which is spread by blood-sucking reduviid insects [1]. It is the leading cause of heart failure in Latin America, with an estimated to 8-10 million people infected [2]. The parasite invades and reproduces in a variety of host cells, including macrophages, smooth and striated muscle, fibroblasts and neurons. Disease progression is marked by an initial acute phase, which typically occurs in children, followed by a symptom-free intermediate phase. A chronic phase leading to GI tract lesions and heart failure often ensues. Current chemotherapy options are limited to nifurtimox and benznidazole, which have been in use since the late 1960s and are compromised by adverse side reactions and low efficacy in chronic disease [3,4]. A need for drugs with more consistent efficacy and less toxicity is manifest.

With an essential requirement for ergosterol [5] and an inability to survive solely on cholesterol salvaged from the host, T. cruzi is vulnerable to inhibitors of the sterol biosynthesis enzyme $14 \alpha-$ demethylase (CYP51) [6,7]. Disruption of CYP51 results in alteration in the ultrastructure of several organelles, decline of endogenous sterols in the parasites, and an accumulation of various $14 \alpha$-methyl sterols with cytostatic and cytotoxic consequences [8]. The broad spectrum antifungal drug posaconazole (Noxafil; Schering-Plough) [9], which targets CYP51, is poised for clinical trials against $T$. cruzi $[6,10,11]$. Posaconazole is capable of inducing parasitological cure in a murine model of both acute and 


\section{Author Summary}

Chagas Disease is caused by kinetoplastid protozoa Trypanosoma cruzi, whose sterols resemble those of fungi, in both composition and biosynthetic pathway. Azole inhibitors of sterol $14 \alpha$-demethylase (CYP51), such as fluconazole, itraconazole, voriconazole, and posaconazole, successfully treat fungal infections in humans. Efforts have been made to translate anti-fungal azoles into a seconduse application for Chagas Disease. Ravuconazole and posaconazole have been recently proposed as candidates for clinical trials with Chagas Disease patients. However, the widespread use of posaconazole for long-term treatment of chronic infections may be limited by hepatic and renal toxicity, a requirement for simultaneous intake of a fatty meal or nutritional supplement to enhance absorption, and cost. To aid our search for structurally and synthetically simple CYP51 inhibitors, we have determined the crystal structures of the CYP51 targets in $T$. cruzi and T. brucei, both bound to the anti-fungal drugs fluconazole or posaconazole. The structures provide a basis for a design of new drugs targeting Chagas Disease, and also make it possible to model the active site characteristics of the highly homologous Leishmania CYP51. This work provides a foundation for rational synthesis of new therapeutic agents targeting the three kinetoplastid parasites.

chronic Chagas Disease, curing between 50-100\% of animals in the acute phase of infection, and $50-60 \%$ of animals chronically infected [7,11]. However, the high manufacturing cost of posaconazole and the requirement for administration via oral suspension simultaneously with a fatty meal or nutritional supplement to enhance absorption may limit its use in treating chronic T. cruzi infections [12]. The search for CYP51-specific compounds that are easier to synthesize and better absorbed upon oral administration continues [13-17].

To rationalize protein-ligand interactions for new inhibitors in T. cruzi, homology modeling based on the x-ray structure of CYP51 from Mycobacterium tuberculosis $\left(\right.$ CYP5 $1_{\text {Mt }}$ ) [18-20] has been used $[14,15,17]$. But CYP $51_{\mathrm{Mt}}$ has only $27 \%$ sequence identity to the T. cruzi enzyme and is unusually exposed to the bulk solvent at the substrate binding site. This structural peculiarity largely excludes the functionally important BC-loop from proteininhibitor interactions and thus limits the utility of $\mathrm{CYP} 5 \mathrm{I}_{\mathrm{Mt}}$ as a model for a Chagas Disease target. The CYP51 BC-loop residue 105 (numbering according to T. cruzi and T. brucei CYP51) is indispensable in the discrimination of the species-specific sterol substrates in T. cruzi and T. brucei [19,21]. Also, a critical mutation hot spot [22], the well conserved BC-loop residue Y116 was reported to be involved in fungal drug resistance, inhibitor binding, and the catalytic function of CYP51 in Candida albicans (Y132, according to C. albicans numbering) [22-27], Histoplasma capsulatum (Y136, according to H. capsulatum numbering) [28], and in the causative agents of zygomycosis in humans, Rhizopus oryzae and Absidia corymbifera [29]. It may therefore play a similar role in T. cruzi.

Here we report the crystal structures for the CYP51 target in $T$. cruzi $\left(\mathrm{CYP} 51_{\mathrm{Tc}}\right.$ ) (resolutions $2.35 \AA$ and $2.27 \AA$ ) and that of the closely related CYP51 ortholog from Trypanosoma brucei $\left(\mathrm{CYP} 51_{\mathrm{Tb}}\right)$ (resolutions $2.7 \AA$ and $2.6 \AA$ ), each bound to an anti-fungal triazole drug, either fluconazole or posaconazole. T. brucei is a protozoan parasite closely related to T. cruzi [30] and the agent of another lethal tropical disease, African Sleeping Sickness. In contrast to T. cruzi and Leishmania spp., it is not clear if the sterol biosynthesis pathway can be targeted in T. brucei. Each parasite has a different life-cycle and different sterol requirements. Although the insect (procyclic) form of T. brucei can undertake de novo sterol biosynthesis, the latter is apparently suppressed in the bloodstream form in the mammalian host, which is supported by receptormediated endocytosis of host low-density lipoproteins that carry phospholipids and cholesterol esters [31]. Nevertheless, CYP51 $1_{\mathrm{Tc}}$ and $\mathrm{CYP} 51_{\mathrm{Tb}}$ do share $83 \%$ sequence identity, a fact which has been crucial for successfully determining their crystal structures and makes it possible to extrapolate structural features learned from one enzyme toward the other. Furthermore, the Leishmania CYP51 are $72-78 \%$ identical to that of $T$. cruzi and T. brucei, so they too can now be modeled to facilitate drug discovery and development.

\section{Materials and Methods}

\section{Design of expression vectors}

By trial-and-error we empirically identified the protein $\mathrm{N}$ terminal modification that eventually led to CYP51 crystals of sufficient quality to determine the x-ray structure. To improve our chances for success, we did the work in parallel on CYP5 1 proteins from Trypanosoma cruzi and Trypanosoma brucei. Five different expression vectors were designed in this work for each CYP51 ortholog to eliminate a stretch of hydrophobic residues which presumably mediate association of the proteins with the endoplasmic reticulum (ER). In their place we introduced hydrophilic or charged sequences at the N-terminus (Table 1). $\mathrm{His}_{6}$-tag $\left(\mathrm{CYP} 51_{\mathrm{Tc}}\right)$ or Hisg-tag $\left(\mathrm{CYP} 51_{\mathrm{Tb}}\right)$ was introduced at the $\mathrm{C}$ terminus to facilitate purification. Coding sequences were subcloned between the NdeI and HindIII restriction cloning sites of the pCWori vector [32] and in this form used to transform Escherichia coli strain HMS174(DE3). The original coding sequence for CYP51 $1_{\mathrm{Tb}}$ contained an internal NdeI site at 345 bp which was silenced by QuickChange site-directed mutagenesis (Stratagene) using forward GGGGTTGGCTATGCTGGG and reverse CCCGAAGGGATAGGACGG PGR primers. DNA amplification reaction: $5 \mathrm{~min}$ at $94^{\circ} \mathrm{C}$, annealing for $1 \mathrm{~min}$ at $50-60^{\circ} \mathrm{C}$, extension for $1.5 \mathrm{~min}$ at $72^{\circ} \mathrm{C}$, for 30 cycles, followed by extension for $10 \mathrm{~min}$ at $72^{\circ} \mathrm{C}$. The highest expression levels were achieved and the best crystals were obtained from the expression constructs modified by replacing the first 21 residues upstream of K22 with the fragment MAKKKKK. Subsequently, based on the analysis of the packing interactions in the crystal, three consecutive glutamate residues, E249-E251, were replaced in $\mathrm{CYP}^{2} 1_{\mathrm{Tb}}$ with alanine by sitedirected mutagenesis (Stratagene) using forward GCGCGGGTGGTGCTGTCAACAAGGACAGC and reverse GCGCGAGGAGGAGGCTTTCGAGCAATGAT PGR primers. DNA amplification reaction: $5 \mathrm{~min}$ at $94^{\circ} \mathrm{C}$, annealing for $1 \mathrm{~min}$ at 45 $65^{\circ} \mathrm{C}$, extension for $1.5 \mathrm{~min}$ at $72^{\circ} \mathrm{C}$, for 35 cycles, followed by extension for $10 \mathrm{~min}$ at $72^{\circ} \mathrm{C}$. This $\mathrm{CYP} 51_{\mathrm{Tb}}$ variant was used to generate the CYP51 $1_{\mathrm{Tb}}$-posaconazole crystals. The identity of all resulting vectors was confirmed by DNA sequencing.

\section{Protein expression and purification}

CYP51 $1_{\text {Tc }}$. One liter of Terrific Broth medium supplemented with $1 \mathrm{mM}$ thiamine, $100 \mu \mathrm{g} / \mathrm{ml}$ ampicillin and trace elements was inoculated with $15 \mathrm{ml}$ of the night culture. Growth continued at $37^{\circ} \mathrm{C}$ and $240 \mathrm{rpm}$ agitation until $\mathrm{OD}_{590}$ reached 1.0. CYP51 $1_{\mathrm{Tc}}$ expression was induced by the addition of isopropyl- $\beta$-D-thiogalactopyranoside (IPTG, final concentration $0.25 \mathrm{mM}$ ) and $\delta$-aminolevulinic acid, a precursor of heme biosynthesis (final concentration $1 \mathrm{mM}$ ). Following induction, temperature was decreased to $28^{\circ} \mathrm{C}$ and agitation to $180 \mathrm{rpm}$. After 40 hours the cells were harvested and lysed by 
Table 1. Design and analysis of the expression vectors.

\begin{tabular}{|c|c|c|c|c|}
\hline \multirow[b]{2}{*}{ Protein } & \multicolumn{2}{|l|}{ Truncation } & \multirow[b]{2}{*}{ Yield } & \multirow[b]{2}{*}{ Crystals } \\
\hline & 110 & 40 & & \\
\hline CYP51 & \multicolumn{2}{|c|}{ MFIEAIVLGLTALILYSVYSVKSFNTTRPTDP PVYPVTVP } & N/A & No \\
\hline CYP51 & MAKKTSSKGKL & PPVYPVTVP & $4 \mathrm{mg} / \mathrm{l}$ & Yes \\
\hline CYP51 & MA & PPVYPVTVP & N/A & No \\
\hline CYP51 & MA & KSFNTTRPTDPPVYPVTVP & N/A & No \\
\hline CYP51 & MAKKKKK & KSFNTTRPTDPPVYPVTVP & 75 mg/l & Yes \\
\hline CYP51 & MAKKKKK & PPVYPVTVP & $5 \mathrm{mg} / \mathrm{l}$ & No \\
\hline CYP51 ${ }_{\mathrm{Tb}} W T$ & \multicolumn{2}{|c|}{ MLLEVAIFLLTALALYSFYFVKSFNVTRPTDPPVYPVTVP } & N/A & No \\
\hline CYP51 Tb $\# 1$ & MAKKTSSKGKL & PPVYPVTVP & $1 \mathrm{mg} / \mathrm{l}$ & No \\
\hline CYP51 $_{\mathrm{Tb}} \# 2$ & MAKKKKK & KSFNTTRPTDPPVYPVTVP & $3 \mathbf{m g} / \mathbf{l}$ & Yes \\
\hline CYP51 $1_{\mathrm{Tb}} \# 3$ & MAKKTSSDEVDEVDEV & DPPVYPVTVP & N/A & No \\
\hline CYP51 ${ }_{\mathrm{Tb}} \# 4$ & MADEVDEVDEV & DPPVYPVTVP & N/A & No \\
\hline CYP51 ${ }_{\mathrm{Tb}} \# 5$ & MA & PPVYPVTVP & N/A & No \\
\hline
\end{tabular}

Highlighted in bold are the constructs which led to the corresponding $\mathrm{x}$-ray structures.

doi:10.1371/journal.pntd.0000651.t001

sonication. Insoluble material was removed from crude extract by centrifugation (45 $\mathrm{min}$ at $35,000 \mathrm{rpm}$ ). The supernatant was subjected to a series of chromatographic steps, including nickelnitrilotriacetic acid (Ni-NTA) agarose (QIAGEN), followed by QSepharose (Amersham Biosciences) in flow-through regime, then by S-Sepharose (Amersham Biosciences). From the S-Sepharose, protein was eluted in a 0.2 to $1.0 \mathrm{M} \mathrm{NaCl}$ gradient and observed by means of $12 \%$ SDS-PAGE to be virtually homogeneous. Fractions containing $\mathrm{P} 450$ were combined, concentrated using a Centriprep concentrating device (Millipore), and stored at $-80^{\circ} \mathrm{C}$. Twenty $\mathrm{mM}$ Tris-HCl, $\mathrm{pH} 8.0,10 \%$ glycerol, $0.5 \mathrm{mM}$ EDTA, and $1 \mathrm{mM}$ DTT were maintained throughout all chromatographic steps.

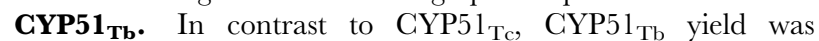
significantly improved via co-expression of the $E$. coli chaperones GroES and GroEL ecoded by the pGro7 plasmid (Takara) cotransformed into the HMS174(DE3) strain. Double transformants were selected on both ampicillin and chloramphenicol containing agar plates. One liter of Terrific Broth medium supplemented with $1 \mathrm{mM}$ thiamine, $100 \mu \mathrm{g} / \mathrm{ml}$ ampicillin, $40 \mu \mathrm{g} / \mathrm{ml}$ chloramphenicol and trace elements was inoculated with $10 \mathrm{ml}$ of the night culture. Growth continued at $37^{\circ} \mathrm{C}$ and $240 \mathrm{rpm}$ agitation until $\mathrm{OD}_{590}$ reached 0.2. Expression of chaperones from the pGro7 vector was induced with $0.2 \%$ arabinose. Growth continued at $26^{\circ} \mathrm{C}$ and $180 \mathrm{rpm}$ until $\mathrm{OD}_{590}$ reached 0.8 . Then CYP $51_{\mathrm{Tb}}$ expression was induced by the addition of isopropyl- $\beta$ D-thiogalactopyranoside (IPTG), final concentration $0.4 \mathrm{mM} ; \delta$ aminolevulinic acid, final concentration $1 \mathrm{mM}$, was also added at this time. Following induction, temperature was decreased to $21^{\circ} \mathrm{C}$. After 44 hours the cells were harvested and lysed by sonication. Purification was conducted as described for CYP5 $1_{\mathrm{Tc}}$.

Both proteins were characterized by UV-vis spectroscopy. For crystallization, protein concentration was determined at $450 \mathrm{~nm}$ from the difference spectra between the CO-bound ferrous and water-bound ferric forms, with an extinction coefficient of $91,000 \mathrm{M}^{-1} \mathrm{~cm}^{-1}$ [33].

\section{Crystallization and data collection}

Screening of crystallization conditions was routinely performed following purification of protein variants using commercial screening kits available in high throughput screening format (Hampton Research), a nanoliter drop-setting Mosquito robot (TTP Labtech) operating with 96-well plates, and a hanging drop crystallization protocol. Optimization of crystallization conditions, if required, was carried out manually in 24-well plates at $23^{\circ} \mathrm{C}$. Proteins were from $1.0-1.8 \mathrm{mM}$ frozen stocks in $20 \mathrm{mM}$ Tris- $\mathrm{HCl}, \mathrm{pH} 7.2\left(\mathrm{CYP} 51_{\mathrm{Tb}}\right)$ or $\mathrm{pH} 8.0\left(\mathrm{CYP}_{51} 1_{\mathrm{Tc}}\right), 10 \%$ glycerol, $0.5 \mathrm{mM}$ EDTA, and $1 \mathrm{mM}$ DDT. The CYP51 $1_{\mathrm{Tb}}$ triple mutant E249A/E250A/E251A was used to obtain CYP51 $1_{\mathrm{Tb}}$-posaconazole crystals. Prior to crystallization proteins were diluted to $0.1-0.2 \mathrm{mM}$ by mixing with $50 \mathrm{mM}$ potassium phosphate at appropriate $\mathrm{pH}$, supplemented with $0.5 \mathrm{mM}$ $\left(\mathrm{CYP} 51_{\mathrm{Tb}}\right)$ or $0.1 \mathrm{mM}\left(\mathrm{CYP}_{\mathrm{Tc}}\right)$ fluconazole. Dilution in the absence of fluconazole or phosphate caused fast precipitation of protein samples. Posaconazole was prepared as $10 \mathrm{mM}$ stock solution in DMSO and has been used at final concentration of $0.2 \mathrm{mM}$. Protein-posaconazole mix was incubated at $4^{\circ} \mathrm{C}$ for one hour prior to crystallization. Crystals of CYP51 $1_{\mathrm{Tb}}$-fluconazole complex grew from $15 \%$ ethylene glycol and $0-3 \%$ acetonitrile. Crystals of $\mathrm{CYP}^{5} 1_{\mathrm{Tb}^{-}}$ posaconazole complex grew from 6\% PEG 4000, 2\% tacsimate, $\mathrm{pH}$ 8.0, and 2\% DMSO. Crystals of $\mathrm{CYP} 51_{\mathrm{Tc}}$-fluconazole grew either from $40 \%$ polypropylene glycol 400 and $0.1 \mathrm{M}$ Tris- $\mathrm{HCl}$, pH 6.0 (PDB ID 2WUZ), or from 25\% PEG 4000 and $0.1 \mathrm{M}$ BisTris, $\mathrm{pH} 5.5$ (PDB ID 2WX2), the latter being harvested directly from the Mosquito 200-nl drop. Prior to data collection, the crystals were cryo-protected by plunging them into a drop of reservoir solution supplemented with 20-24\% ethylene glycol or 20\% glycerol, and flash-frozen in liquid nitrogen.

All native and two-wavelength anomalous dispersion $\mathrm{x}$-ray diffraction data were collected at $100-110 \mathrm{~K}$ at beamline 8.3.1, Advanced Light Source, Lawrence Berkeley National Laboratory, USA. Anomalous diffraction data were collected from one CYP5 $11_{\mathrm{Tb}}$ crystal at two wavelengths, one corresponding to the median between the Fe peak and the inflection point and the other at $375 \mathrm{eV}$ higher (Table 2). Data indexing, integration, scaling, phasing, and density modification were conducted using the ELVES automated software suite [34] (Tables 2 and 3).

\section{Structure determination and refinement}

CYP $51_{\mathrm{Tb}}$-fluconazole data processed in $\mathrm{P} 3{ }_{1} 21$ with $\mathrm{R}_{\text {merge }}$ of $6.5 \%$ allowed for location of a single $\mathrm{Fe}$ atom. Initial phases with an overall figure of merit of 0.26 were improved by solvent flattening (mean figure of merit 0.85 after solvent flattening) to provide an interpretable electron density map (Table 2). Automated model 
Table 2. Fe anomalous dispersion data collection and phasing statistics.

\begin{tabular}{|c|c|c|}
\hline Protein & \multicolumn{2}{|l|}{ CYP51 } \\
\hline Ligand & \multicolumn{2}{|l|}{ Fluconazole } \\
\hline \multicolumn{3}{|l|}{ Data collection } \\
\hline Space group & \multicolumn{2}{|l|}{$P 3,21$} \\
\hline \multicolumn{3}{|l|}{ Cell dimensions } \\
\hline$a, b, c(\AA)$ & \multicolumn{2}{|c|}{$106.4,106.4,99.8$} \\
\hline$\alpha, \beta, \gamma\left({ }^{\circ}\right)$ & \multicolumn{2}{|l|}{$90,90,120$} \\
\hline \multirow[t]{2}{*}{ Molecules in AU } & \multicolumn{2}{|l|}{1} \\
\hline & Peak/Inflection & Remote \\
\hline Wavelength & 1.7393 & 1.6531 \\
\hline Resolution $(\AA)$ & 3.2 & 3.2 \\
\hline$R_{\text {sym }}$ or $R_{\text {merge }}(\%)$ & $6.5(-)^{1}$ & $6.2(-)$ \\
\hline$|/ \sigma|$ & $21.1(2.3)$ & $22.4(2.9)$ \\
\hline Completeness (\%) & $100(93.4)$ & $100(99.1)$ \\
\hline Redundancy & $18.8(9.5)$ & $20.2(13.0)$ \\
\hline \multicolumn{3}{|l|}{ Phasing } \\
\hline Resolution range & \multicolumn{2}{|l|}{$3.2-67.7$} \\
\hline No. of used sites & \multicolumn{2}{|l|}{1} \\
\hline Phasing power & 0.38 & 0.36 \\
\hline Figure of merit & \multicolumn{2}{|l|}{0.26} \\
\hline After density modification & \multicolumn{2}{|l|}{0.85} \\
\hline
\end{tabular}

building using BUCCANEER [35] placed the polyalanine backbone for $84 \%$ of the residues in the asymmetric unit. The remaining residues were built manually with COOT [36], alternated with TLS and positional refinement using REFMAC [37,38]. The structure was refined to $3.2 \AA$ with the $\mathrm{R}$ and $\mathrm{R}_{\text {free }}$ values of $32.0 \%$ and $38.0 \%$, respectively. Although showing up largely as a polypeptide backbone at low resolution, this $\mathcal{T}$. brucei structure served as a search model for molecular replacement in determining the $\mathrm{x}$-ray structure for CYP5 $1_{\mathrm{Tc}_{\mathrm{c}}}$ using $2.35 \AA$ native data processed as $\mathrm{P} 21_{1}$ with $\mathrm{R}_{\text {merge }}$ of $11 \%$. Two CYP $51_{\mathrm{Tc}}$ molecules were placed in an asymmetric unit. Manual model building with COOT [36] alternated with TLS and positional refinement using REFMAC [37,38] resulted in the final CYP $51_{\mathrm{Tc}}$ structure with the $\mathrm{R}$ and $\mathrm{R}_{\text {free }}=$ values of $21.7 \%$ and $27.5 \%$ and the Ramachandran statistics of $93.8 \%$ residues in preferred regions, $5.2 \%$ in allowed regions, and $1 \%$ (9 residues) outliers, as calculated by COOT. NCS restrains were applied at all stages of the refinement. The refined CYP5 $1_{\mathrm{Tc}}$ structure was used as a starting model against both the $2.27 \AA$ native data for CYP5 $1_{\mathrm{Tc}_{\mathrm{c}}}$ and $2.7 \AA$ native data for $\mathrm{CYP} 51_{\mathrm{Tb}}$, which allowed the majority of the CYP51 1 Tb side chains to be built in. At that time, refinement of CYP $51_{\mathrm{Tb}}$ converged with $\mathrm{R}$ and $\mathrm{R}_{\text {free }}$ of $21.0 \%$ and $27.4 \%$, respectively. Ramachandran statistics indicate $91.2 \%$ residues in preferred regions, $5.9 \%$ in allowed regions, and 2.9\% (13 residues) outliers. Refinement of $2.27 \AA$ CYP5 $1_{\mathrm{Tc}}$ data converged with $\mathrm{R}$ and $\mathrm{R}_{\text {free }}$ of $19.3 \%$ and $27.3 \%$, respectively, and the Ramachandran statistics of $95.6 \%$ residues in preferred regions, $3.5 \%$ in allowed regions, and $0.9 \%$ (9 residues) outliers.

Analysis of the crystallographic symmetry packing interactions in the $\mathrm{CYP} 51_{\mathrm{Tb}}$-fluconazole complex revealed contacts between the triplets of glutamate residues D249-D251 situated in the GH- loop. To reduce electrostatic repulsion, all three residues were replaced with alanine. Although this modification did not improve resolution of the CYP5 $1_{\mathrm{Tb}}$-fluconazole crystals, the triple mutant was more amenable to co-crystallization with posaconazole. The CYP5 $1_{\mathrm{Tb}}$ coordinates refined to $2.7 \AA$ served as a search model to determine $\mathrm{CYP} 51_{\mathrm{Tb}}$-posaconazole structure using $2.6 \AA$ native data processed as $\mathrm{C} 2$ with $\mathrm{R}_{\text {merge }}$ of $8.5 \%$. Four protein molecules were placed in an asymmetric unit. Refinement converged with $\mathrm{R}$ and $\mathrm{R}_{\text {free }}$ of $19.1 \%$ and $26.4 \%$, respectively and the Ramachandran statistics of $95.3 \%$ residues in preferred regions, $4.0 \%$ in allowed regions, and $0.7 \%$ (13 residues) outliers. In all structures, side chains not visible in the density were modeled as alanine (Table 3).

\section{Molecular docking}

Binding of posaconazole to $\mathrm{CYP} 51_{\mathrm{Tc}}$ was predicted by molecular docking using the 2WUZ structure. Docking was carried out using GLIDE (version 5.0) [39]. The docking protocol was validated by re-docking of fluconazole, which reproduced the binding mode observed in the crystal structure. The protein was initially prepared by the Protein Preparation Wizard module using default options. Hydrogen atoms were added to the complex structure, followed by a restrained minimization using the OPLS2005 force field. The Receptor Grid Generation module was then employed to prepare a rigid receptor grid centered at M360, which contains the entire binding tunnel of the energy minimized complex, for subsequent docking. The three-dimensional structure of posaconazole was generated by the Ligprep module with the OPLS2005 force field. Computational docking was performed using GLIDE in standard precision (SP) mode, and binding affinities were estimated as GLIDE score. The characteristic coordination between the heme group and the ligand was modeled by applying a constraint at the $\mathrm{Fe}^{3+}$ ion of the heme group that imposed interaction with one of the nitrogen atoms from the ligand's triazolyl ring. Since posaconazole (molecular weight $=700.8 \mathrm{~g} / \mathrm{mol}$ ) is significantly larger and longer than fluconazole (molecular weight $=306.3 \mathrm{~g} / \mathrm{mol}$ ) (Fig. 1), the van der Waals radii of the ligand were softened by a scaling factor of 0.6 in the initial docking calculation, which predicted two binding poses with similar Glide scores of -9.23 and -9.60 . The binding model was further refined by relaxing the binding tunnel in the presence of posaconazole. Side chains of residues within $4 \AA$ from the docked posaconazole were optimized by performing side-chain refinement with Prime (version 2.0) [40]. The resulting complex was used to re-dock posaconazole with van der Waals radii scaled by the default value of 0.8 . Consistent with the initial calculations, the second-round docking also predicted the same binding orientations with favorable and similar GLIDE scores (pose $1=-11.07$; pose $2=-10.70$ ).

\section{Accession codes}

Protein Data Bank: coordinates and structure factors have been deposited with accession codes 2WUZ, 2WX2, 2WV2 and 2X2N.

\section{Results and Discussion}

Protein design and determination of the $x$-ray structures

By trial-and-error, the highest expression levels and best crystals for both $\mathrm{CYP} 51_{\mathrm{Tc}}$ and $\mathrm{CYP} 51_{\mathrm{Tb}}$ were obtained from the expression constructs modified by replacing the first 21 residues upstream of K22 with the highly positively charged fragment MAKKKKK (Table 1). The triple E249A/E250A/E251A $\mathrm{CYP} 1_{\mathrm{Tb}}$ mutant was based upon this N-terminally modified construct. The UV-vis spectra of purified proteins revealed 
Table 3. Data collection and refinement statistics.

\begin{tabular}{|c|c|c|c|c|}
\hline \multirow[b]{2}{*}{ Protein } & \multicolumn{3}{|l|}{ Native } & \multirow[b]{2}{*}{ CYP51 } \\
\hline & \multirow{2}{*}{$\frac{\text { CYP51TC }_{\text {Fluconazole }}}{\text { Flucon }}$} & \multirow{2}{*}{$\frac{\text { CYP51Tc }}{\text { Fluconazole }}$} & \multirow{2}{*}{$\frac{\text { CYP51 }_{\mathrm{Tb}}}{\text { Fluconazole }}$} & \\
\hline Ligand & & & & Posaconazole \\
\hline PDB ID & $2 W \times 2$ & 2WUZ & $2 W V 2$ & $2 \times 2 N$ \\
\hline \multicolumn{5}{|l|}{ Data collection } \\
\hline Space group & $\mathrm{P} 2_{1}$ & $\mathrm{P} 2_{1}$ & $P 3,21$ & $\mathrm{C} 2$ \\
\hline \multicolumn{5}{|l|}{ Cell dimensions } \\
\hline$a, b, c(\AA)$ & $70.0,101.5,74.7$ & $74.9,92.6,78.3$ & $106.2,106.2,99.7$ & $199.9,114.5,138.1$ \\
\hline$\alpha, \beta, \gamma\left({ }^{\circ}\right)$ & $90,111.63,90$ & $90,102.1,90$ & $90,90,120$ & $90,131.8,90$ \\
\hline Molecules in AU & 2 & 2 & 1 & 4 \\
\hline Wavelength & 1.1159 & 1.1159 & 1.1159 & 1.1159 \\
\hline Resolution $(\AA)$ & 2.27 & 2.35 & 2.7 & 2.6 \\
\hline$R_{\text {sym }}$ or $R_{\text {merge }}(\%)$ & $9.0(42.0)^{1}$ & $11.0(50.0)$ & $10.7(-)^{2}$ & $8.5(76.9)$ \\
\hline$|/ \sigma|$ & $8.5(2.4)$ & $7.4(1.5)$ & $17.0(2.8)$ & $9.2(1.7)$ \\
\hline Completeness (\%) & $100.0(55.7)$ & $93.6(70.8)$ & $100.0(100.0)$ & $99.8(100.0)$ \\
\hline Redundancy & $3.6(2.4)$ & $3.4(2.4)$ & $44.1(44.5)$ & $3.9(3.9)$ \\
\hline \multicolumn{5}{|l|}{ Phasing } \\
\hline \multicolumn{5}{|l|}{ Resolution range } \\
\hline \multicolumn{5}{|l|}{ No. of used sites } \\
\hline \multicolumn{5}{|l|}{ Phasing power } \\
\hline \multicolumn{5}{|l|}{ Figure of merit } \\
\hline \multicolumn{5}{|c|}{ After density modification } \\
\hline \multicolumn{5}{|l|}{ Refinement } \\
\hline No. reflections & 38067 & 38792 & 17043 & 71168 \\
\hline$R_{\text {work }} / R_{\text {free }}(\%)$ & $19.3 / 27.3$ & $21.7 / 27.5$ & $21.0 / 27.4$ & $19.1 / 26.4$ \\
\hline \multicolumn{5}{|l|}{ No. atoms } \\
\hline Protein & 6954 & 6994 & 3433 & 14048 \\
\hline Heme & 86 & 86 & 43 & 172 \\
\hline Ligand & 44 & 44 & 22 & 204 \\
\hline Water & 429 & 179 & 15 & 253 \\
\hline Mean B value & 23.7 & 44.6 & 45.8 & 53.9 \\
\hline \multicolumn{5}{|l|}{$B$-factors } \\
\hline Protein & 23.8 & 44.9 & 37.2 & 54.2 \\
\hline Heme & 15.5 & 41.6 & 65.0 & 47.7 \\
\hline Ligand & 32.1 & 47.0 & 93.1 & 50.7 \\
\hline Water & 25.3 & 43.5 & 36.6 & 46.4 \\
\hline \multicolumn{5}{|l|}{ R.m.s deviations } \\
\hline Bond lengths $(\AA)$ & 0.016 & 0.019 & 0.018 & 0.016 \\
\hline Bond angles $\left({ }^{\circ}\right)$ & 1.7 & 1.9 & 1.8 & 1.7 \\
\hline
\end{tabular}

${ }^{1}$ Values in parentheses are for highest-resolution shell.

${ }^{2} R_{\text {sym }}$ is meaningless when the individual spot $I / \sigma l$ value is below 1 .

doi:10.1371/journal.pntd.0000651.t003

features characteristic for homogeneous and normally folded P450 (Fig. 2). We first determined the crystal structure for CYP5 $1_{\mathrm{Tb}}$ using anomalous dispersion of the heme iron. Although largely a backbone trace at $3.2 \AA$ resolution, this structure served as a search model for molecular replacement in determining the CYP51 $1_{\mathrm{Tc}}$-fluconazole structure at $2.35 \AA$, which was used as a search model against the $2.27 \AA \mathrm{CYP}^{2} 1_{\mathrm{Tc}}$-fluconazole data to reveal an alternative conformation of fluconazole bound in the active site. This same CYP51 $1_{\mathrm{Tc}}$ structure was used as a model against the $2.7 \AA$ CYP $51_{\mathrm{Tb}}$-fluconazole data. Refined to $2.7 \AA$ CYP51 $1_{\mathrm{Tb}}$ coordinates subsequently served as a search model for determining the $2.6 \AA \mathrm{CYP} 51_{\mathrm{Tb}}$-posaconazole structure.

\section{Overall crystal structures of CYP51}

CYP51 $1_{\mathrm{Tc}}$ and $\mathrm{CYP} 51_{\mathrm{Tb}}$ have a common $\mathrm{P} 450$ protein fold characterized by the sets of the $\alpha$-helices and $\beta$-sheets highlighted in Fig. 3A, B. The T. cruzi and T. brucei structures superimpose with r.m.s.d. of $0.89 \AA$ for $\mathrm{C} \alpha$ atoms, with the most pronounced 


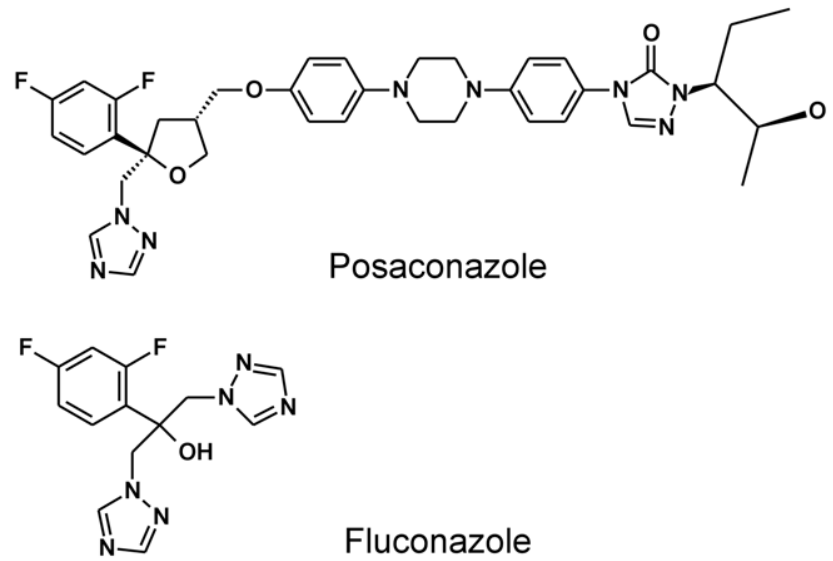

Figure 1. Chemical structures of posaconazole and fluconazole. doi:10.1371/journal.pntd.0000651.g001

differences in the region encompassing the $\mathrm{F}$ and $\mathrm{G}$ helices and the loop between them (Fig. 4A). By contrast, Trypanosoma CYP51 enzymes do not superimpose nearly as well with bacterial CYP51 $1_{\mathrm{Mt}}$ (r.m.s.d. of $1.83 \AA$ ) (Fig. 4B), being more similar to their human counterpart $\left(\mathrm{CYP} 5 \mathrm{l}_{\mathrm{h}}\right)$, based on both backbone similarity (r.m.s.d. of $1.45 \AA$ ) and solvent exposure at the active site (Fig. 4C). All three eukaryotic enzymes lack the extreme bending of the I-helix that is associated with CYP5 $1_{\mathrm{Mt}}$, resulting in their active sites being more isolated from the bulk solvent. The structured BC-region in Trypanosoma CYP51 includes the B'-helix encompassed by the short $\eta$-helices blocking access to the active site from the bulk solvent. Seven residues from the BC-region, V102, Y103, I105, M106, F110, A115 and Y116, are part of the active site in CYP5 $1_{\mathrm{Tc}}$ and $\mathrm{CYP} 51_{\mathrm{Tb}}$, which is consistent with our previous observation that a series of CYP51 inhibitors reported elsewhere [16] have higher binding affinities toward Trypanosoma CYP51 compared to CYP51 1 , where these residues do not participate in the active site due to the "open" conformation of the loop. The two CYP51 $1_{\mathrm{Tc}}$-fluconazole structures reported here superimpose with r.m.s.d of $0.68 \AA$, revealing some conformational differences in the F-helix and the BC-loop, which may account for the distinct fluconazole binding modes and result in repacking of protein molecules in the crystal lattice (Table 3).

In the CYP5 $1_{\mathrm{Tb}}$-posaconazole structure, four protein molecules in the asymmetric unit superimpose with the r.m.s.d. within of $0.5 \AA$, revealing virtually no conformational variations. However, posaconazole samples two distinct conformations due to the long tail swinging $\sim 7-8 \AA$ in the hydrophobic mouth of the substrate binding tunnel (Fig. 3B). The entrance to the tunnel is marked by a patch of the hydrophobic residues (colored yellow in Fig. 3B), which apparently guide access of the sterol substrates to the active site.

\section{Fluconazole binding in the active site}

As expected, fluconazole is bound in the active site by coordination to the heme iron via the aromatic nitrogen atom of a triazole ring and by multiple van der Waals and aromatic stacking interactions (Fig. 5). All residues within $7 \AA$ of fluconazole (Fig. 6A) are labeled with blue triangles in Fig. 7. The 2,4difluorophenyl moiety is enclosed in the pocket formed by the heme macrocycle, the aromatic side chains of Y103, F110, Y116 (BC-loop) and F290 (I-helix), and aliphatic side chains M106, A287 and A291. Although fluconazole occupies the same pocket in both CYP51 $1_{\mathrm{Tc}}$ structures, it adopts two conformations that differ by the $180^{\circ}$ flipping of the 2,4-difluorophenyl moiety. Orientation 1 is observed both in the $2.27 \AA \mathrm{CYP}^{2} 1_{\mathrm{Tc}^{-}}$ fluconazole structure reported in this work (PDB ID 2WX2) (Fig. 5A) and in the CYP51 $1_{\mathrm{Mt}}$-fluconazole complex reported elsewhere (PDB ID 1EAl) [18]. The same conformation is adopted by the 2,4-difluorophenyl ring of posaconazole in the CYP51 $1_{\mathrm{Tb}}$-posaconazole complex in all four molecules in the asymmetric unit. In orientation 1, Y103 makes a $2.7 \AA$ H-bonding contact to the main chain amide group of M360. A $180^{\circ}$ flipped orientation of the ring, orientation 2, is observed in the $2.35 \AA$ GYP51 $1_{\mathrm{Tc}}$-fluconazole structure (PDB ID 2WUZ) (Fig. 5B). As evidenced by the residual $\mathrm{F}_{\mathrm{O}}-\mathrm{F}_{\mathrm{C}}$ electron density map calculated for
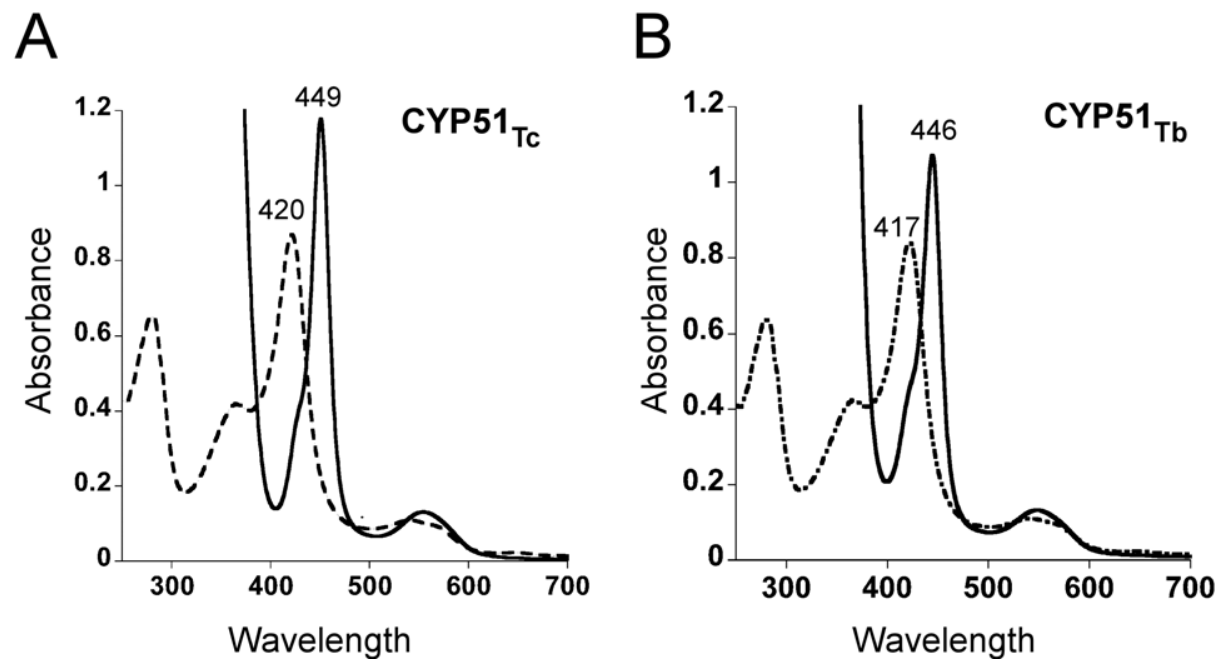

Figure 2. Spectral characterization of CYP51 ${ }_{\mathrm{Tc}}$ and CYP51 $1_{\mathrm{Tb}}$. Soret and visible regions of the CYP51 $1_{\mathrm{Tc}}(\mathbf{A})$ and $\mathrm{CYP} 51_{\mathrm{Tb}}(\mathbf{B})$ spectra are shown. The ferric protein (dashed trace) was reduced with sodium dithionite to a ferrous form (solid trace) in the presence of CO. The spectra were recorded at room temperature in a $1 \mathrm{ml}$ quartz cuvette containing $1 \mu \mathrm{M}$ CYP51 in $10 \mathrm{mM}$ Tris- $\mathrm{HCl}, \mathrm{pH}$ 7.5, and 10\% glycerol using a Cary UV-visible scanning spectrophotometer (Varian). CYP51 $\mathrm{Tc}$ has a Soret maximum at $420 \mathrm{~nm}$ which upon reduction with sodium dithionite and CO binding shifts to $449 \mathrm{~nm}$ (A). CYP51 $\mathrm{Tb}$ has a Soret maximum at $417 \mathrm{~nm}$ which upon reduction and CO binding shifts to $446 \mathrm{~nm}$ (B).

doi:10.1371/journal.pntd.0000651.g002 


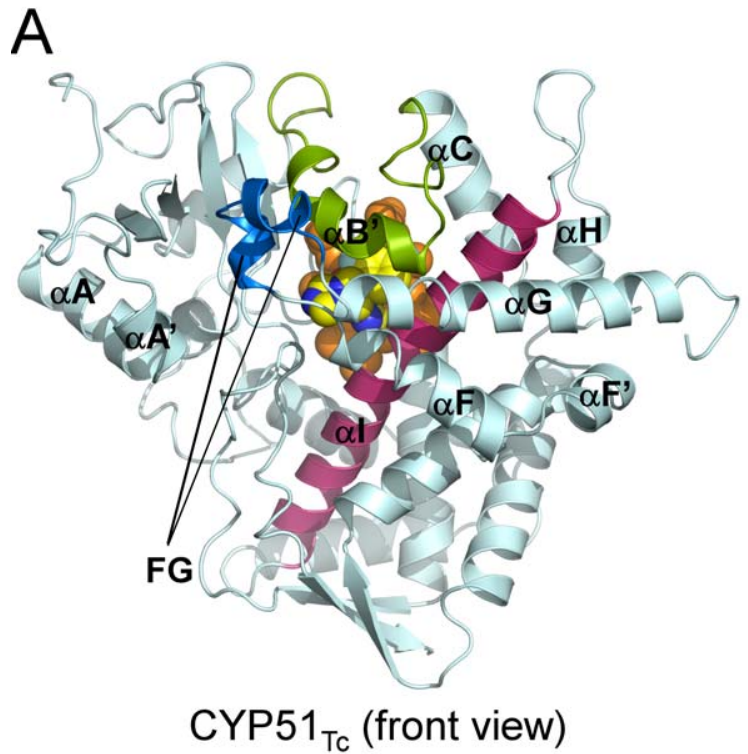

B

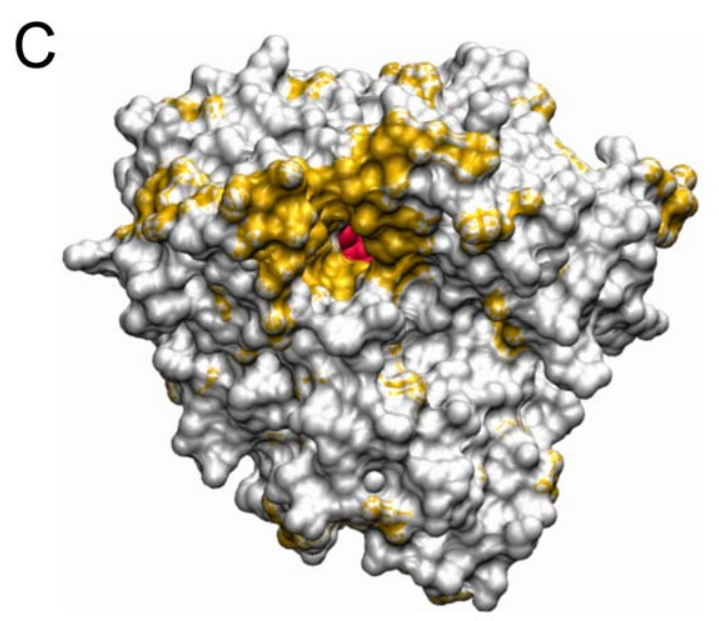

CYP51 $_{\text {Tb }}$ (front view)

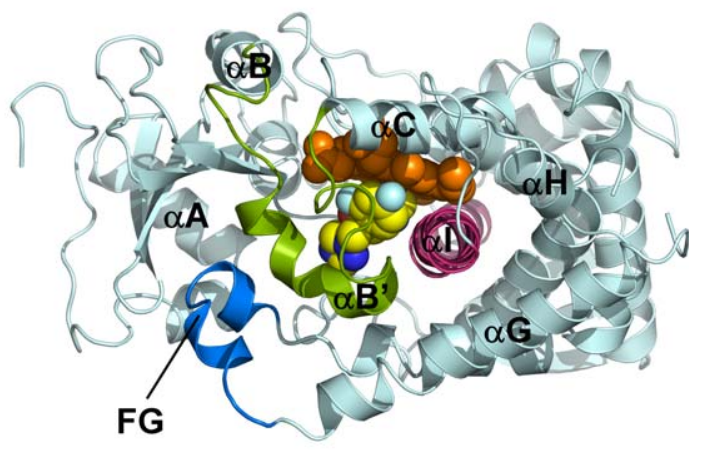

CYP51 1 Tc $($ top view)

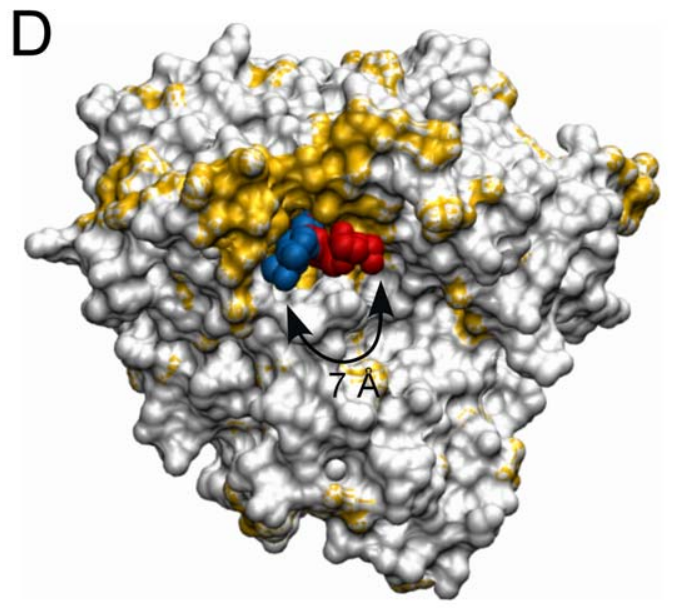

CYP51 ${ }_{\mathrm{Tb}}$-posaconazole

Figure 3. Overall structures of CYP51. A, B. Fluconazole-bound CYP51 $1_{\mathrm{Tc}}$ with selected $\alpha$-helices labeled (PDB ID Code 2 WUZ). A. Distal protein surface with respect to heme. B. Image is rotated $\sim 90^{\circ}$ toward viewer. Protein backbone is depicted by cyan ribbon with the I-helix highlighted in magenta, BC-region in green, FG-region in blue. Heme (orange) and fluconazole are depicted by spheres. Fluconazole color scheme: carbon yellow, oxygen red, nitrogen blue, fluorine cyan. Images were prepared using PYMOL [58] unless indicated otherwise. C, D. CYP51 $1_{\mathrm{Tb}}$ in surface representation with the hydrophobic residues colored in golden yellow. In C, posaconazole is omitted for clarity, heme prosthetic group (pink) shows through the hydrophobic tunnel entrance. In $\mathbf{D}$, two overlaid posaconazole conformers are shown protruding out of the tunnel opening. Bent conformer (chain B) is in red, extended conformer (chain D) is in blue. Images were generated using VMD program [59].

doi:10.1371/journal.pntd.0000651.g003

the orientation 1 (pink mesh in Fig. 5B), the 2-fluoro substituent of the fluconazole difluorophenyl ring in $2 \mathrm{WUZ}$ must point toward the heme macrocycle. A 2.6 $\AA$ H-bonding contact between the 2fluoro substituent and the hydroxyl group of Y103 may help to stabilize orientation 2, which appears to be less sterically favorable than orientation 1 .

Perhaps both ring conformations co-exist in the CYP51 $\mathrm{Tc}^{-}$ fluconazole complex, possibly correlated with the conformation of the BC-loop which affects H-bonding pattern of Y103. In orientation 1, the H-bond between the 2-fluoro substituent and Y103 is broken due to the $3.5 \AA$ reorientation of Y103 toward M360 resulting in the $2.7 \AA \mathrm{H}$-bond to its amide nitrogen and in the flipping of the 2,4-difluorophenyl ring into a sterically more favorable orientation with the fluorinated edge facing away from the heme macrocycle (Fig. 5B). Crystallization conditions may have served to shift the equilibrium by stabilizing one of these states. The entire fluconazole molecule is shifted about $1.5 \AA$ between the two CYP51 $1_{\mathrm{Tc}}$ structures, which may be related to the low efficacy of this drug against T. cruzi. Given that the CYP5 $1_{\mathrm{Tb}}$ active site is virtually identical to that of $T$. cruzi, the same equilibrium would be expected to occur in T. brucei. However, we could not observe this phenomenon as $\mathrm{CYP} 51_{\mathrm{Tb}}$-fluconazole complex has been co-crystallized under a single set of conditions with one molecule in the asymmetric unit.

\section{Hydrophobic tunnel}

The CYP5 $1_{\text {Tc }}$ structures revealed a 42 residue-long hydrophobic tunnel connecting the chamber adjacent to the heme with the 

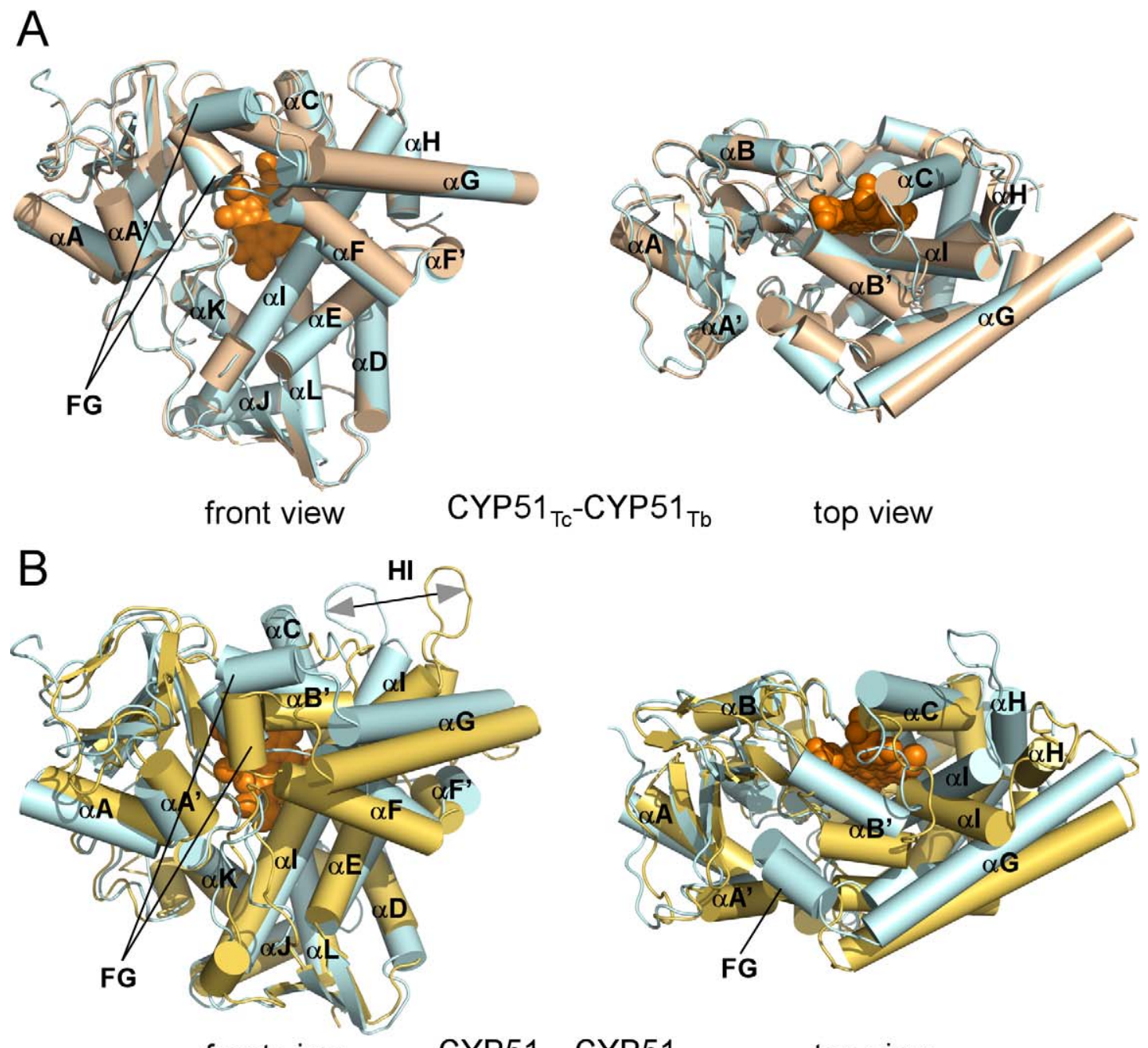

front view

$\mathrm{CYP}^{5} 1_{\mathrm{Tc}}-\mathrm{CYP} 51_{\mathrm{Mt}}$

top view

C
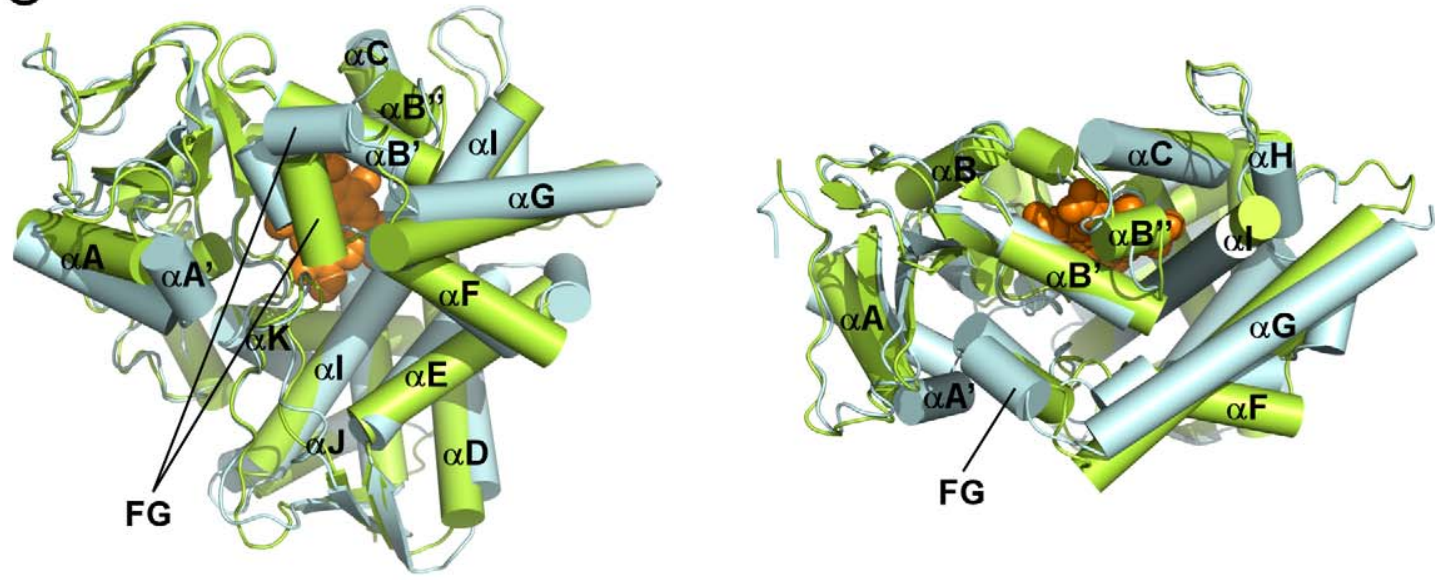

front view

$\mathrm{CYP}^{5} 1_{\mathrm{Tc}}-\mathrm{CYP} 51_{\mathrm{h}}$

top view

Figure 4. Comparison between CYP51 from different phyla. A. CYP51 $1_{\mathrm{TC}}$ (cyan in A, B and C, PDB ID Code 2WUZ) and CYP51 $1_{\mathrm{Tb}}(\mathrm{Wheat}, \mathrm{PDB} I D$ Code 2WV2) superimposed with r.m.s.d. of $0.89 \AA$ A. Helices are represented by labeled cylinders. Fluconazole is omitted for clarity. B. CYP51 CYP51 $1_{\mathrm{Mt}}$ (golden yellow, PDB ID Code 2VKU) superimposed with r.m.s.d of $1.83 \AA$. C. CYP51 $1_{\mathrm{Tc}}$ and $\mathrm{CYP5} 1_{\mathrm{h}}$ (lemon green, PDB ID Code: $313 \mathrm{~K}$ ) superimposed with r.m.s.d of $1.45 \AA$. In each panel, distal surface is shown on the right. Image on the left is rotated $\sim 90^{\circ}$ toward viewer. doi:10.1371/journal.pntd.0000651.g004 

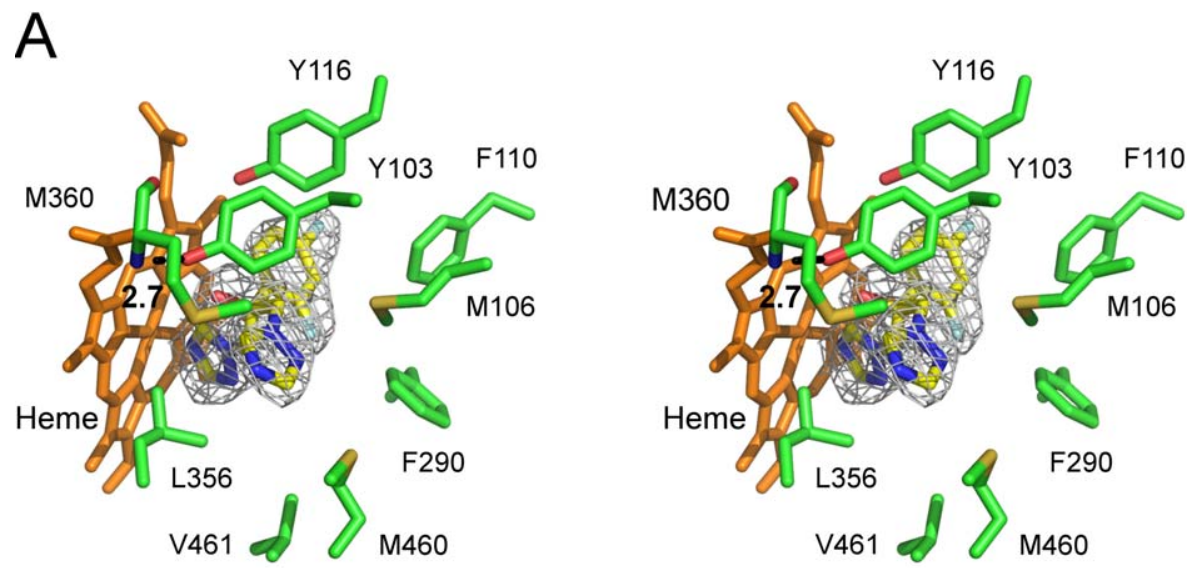

Fluconazole orientation 1 (PDB ID 2WX2)
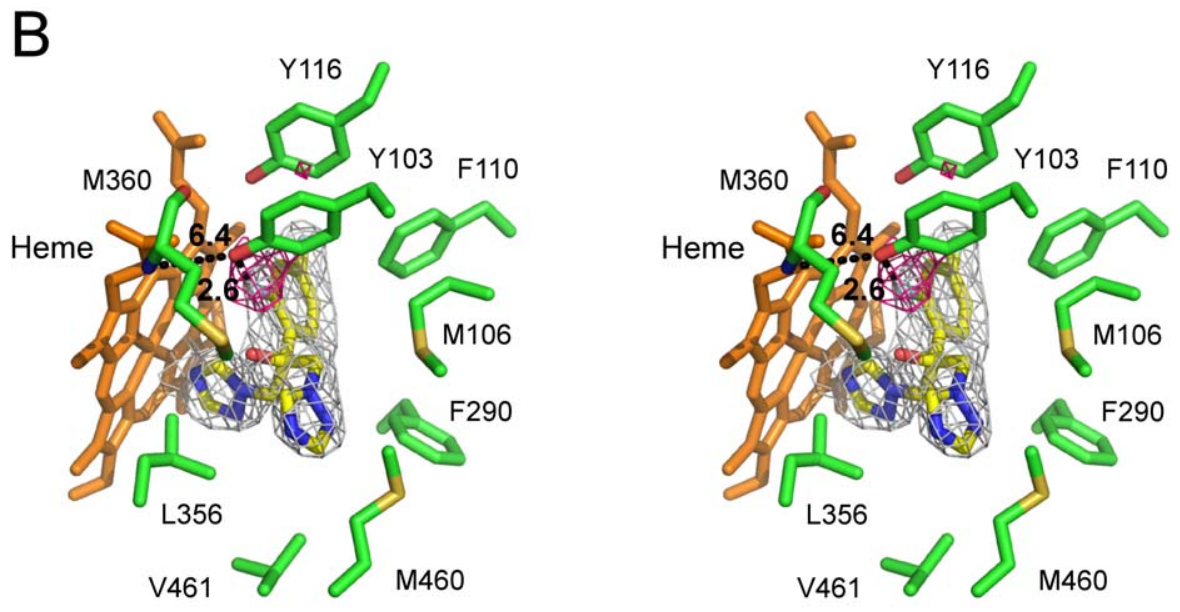

Fluconazole orientation 2 (PDB ID 2WUZ)

Figure 5. Fluconazole binding in CYP51 $1_{\mathrm{Tc}}$ A, B. Stereoscopic view of CYP51 $1_{\mathrm{Tc}}$ with fluconazole bound in active site. Side chains of the residues within 4 A of fluconazole are in green. For clarity, A287, A291 and T295 are omitted. Main chain atoms are shown for M360. Fluconazole color scheme as in Fig. 3. Fragments of $2 \mathrm{~F}_{\mathrm{o}}-\mathrm{F}_{\mathrm{c}}$ electron density map calculated with the fluconazole coordinates omitted from the input file are shown as grey wire mesh. Chain $\mathrm{A}$ has been used in both structures to generate the images. A. Fluconazole orientation 1 in $2 \mathrm{WX} 2$ structure; $\mathrm{OH}$-group of $\mathrm{Y} 103 \mathrm{H}$-bonds to the amide nitrogen of M360. B. Fluconazole orientation 2 in the 2 WUZ structure; peak in the positive $F_{o}-F_{c}$ map (pink mesh) calculated with 2,4difluorophenyl ring in flipped orientation superimposes with the 2-fluorine $\mathrm{H}$-bonding to $\mathrm{Y} 103$.

doi:10.1371/journal.pntd.0000651.g005

protein surface (Fig. 6B). Residues constituting the tunnel in addition to those interacting with fluconazole are labeled with green triangles in Fig. 7. The mouth of the channel is surrounded by residues I45, I46, G49, K50, I209, P210, H458, and M460, which may delineate the substrate/inhibitor entry site in eukaryotic CYP51 (Fig. 8). This entry mode would be in contrast to that in CYP5 $1_{\mathrm{Mt}}$, where it most likely occurs through the open BC-loop. The tunnel-forming residues are invariant between CYP51 $1_{\mathrm{Tc}}$ and $\mathrm{CYP} 51_{\mathrm{Tb}}$ with the exception of four conservative substitutions at positions 46, 105, 215 and 359. The residue at position 105 (highlighted cyan in Fig. 7) is known to dominate substrate specificity with respect to the methylation status of the C4 atom in CYP51 sterol substrates. I105 in T. cruzi allows efficient conversion of C4-dimethylated 24-methylenedihydrolanosterol while the bulkier F105 in T. brucei favors C4-monomethylated norlanosterol [19,21]. Phenylalanine in $\mathrm{CYP}_{\mathrm{Tb}}$ protrudes further into the active site than isoleucine in $\mathrm{CYP}_{\mathrm{T}} \mathrm{1}_{\mathrm{Tc}}$ (Fig. 6A), potentially resulting in interference with the $4 \beta$-methyl group of the sterol substrate. However, F105 does not interfere with either posaconazole or fluconazole binding.

Comparison of the residues constituting the tunnel in CYP5 $1_{\mathrm{Tc}}$ with the human counterpart, CYP5 $1_{h}$, indicates that two residues, H236 and H489 (numbered according to the human sequence and highlighted yellow in Fig. 7), protrude into the tunnel near the opening, reducing both its size and hydrophobicity. As they are present exclusively in mammalian orthologues [41], H236 and H489 may partly account for the selectivity of azole drugs toward pathogenic fungi and protozoa. In accord with this hypothesis, proline corresponding to $\mathrm{H} 236$ in pathogenic fungi is among hot spots that confer resistance to posaconazole in Aspergillus fumigatus (P216) [42] and Candida albicans (P230) [43].

\section{Posaconazole binding}

The hydrophobic tunnel in $\mathrm{CYP} 51_{\mathrm{Tb}}$ accommodates the antifungal drug posaconazole in either extended or bent conformations. The $2.6 \AA$ structure of the CYP $51_{\mathrm{Tb}}$-posaconazole 

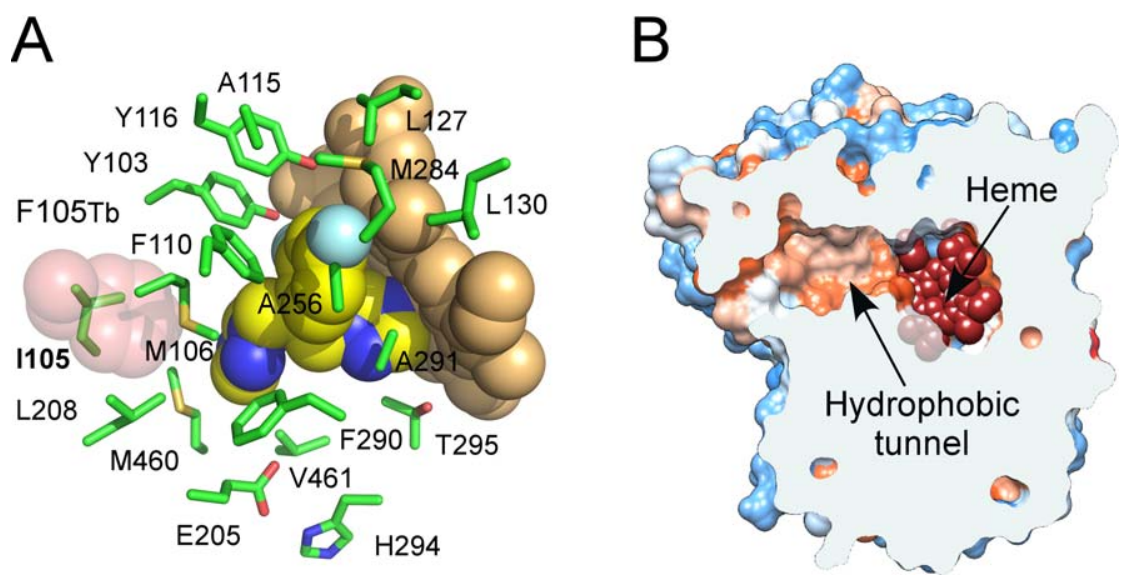

Figure 6. Hydrophobic tunnel. A. Fluconazole in orientation 2 bound to CYP51 $1_{\text {Tc. }}$ Fluorinated edge of the 2,4-difluorophenyl ring faces heme macrocycle. Fluconazole and heme are shown as van der Waals spheres; residues within $7 \AA$ of fluconazole as sticks. Color scheme for heme and fluconazole as in Fig. 3. F105 superimposed from CYP51 $1_{\mathrm{Tb}}$ is shown as semitransparent pink spheres. B. Front view of CYP51 $\mathrm{Tc}$ clipped by plane (cyan) through substrate binding tunnel. Hydrophobic areas are orange, hydrophilic areas blue. Heme at end of tunnel: with van der Waals spheres in red. Fluconazole is removed for clarity. Image was prepared using CHIMERA [60].

doi:10.1371/journal.pntd.0000651.g006

complex revealed four protein monomers in an asymmetric unit with posaconazole coordinating to the heme iron in a manner similar to that of fluconazole with the fluorinated edge of the 2,4difluorophenyl ring facing away from the heme macrocycle, and the long substituent tail extending into the hydrophobic tunnel. Electron density is well defined for the Fe-coordinating head of the posaconazole molecule in all four monomers but somewhat fades out toward its long tail (Fig. 8A). Thus, the terminal 2hydroxypentan group is defined in none of four monomers. Three from the four monomers (chains A, B, and C) accommodate posaconazole in bent conformation while in the monomer $\mathrm{D}$ posaconazole is in extended conformation. Conformational variability of posaconazole is enabled by the interconversion of the piperazine six-membered ring between the chair and twisted boat conformations. The latter serves to accommodate the bend. Electron density is best defined in monomer B, where the terminal phenyl-2-hydroxypentan-triazolone group of posaconazole lies within $6 \AA$ of protein residues I209-P210-A211 and V213-F214 which are invariant between $\mathrm{CYP} 51_{\mathrm{Tc}}$ and $\mathrm{CYP} 51_{\mathrm{Tb}}$. P210, the mutation hot spot in fungi, is situated right in the bend of the posaconazole molecule (Fig. 9A). In the extended conformation in monomer $\mathrm{D}$, the phenyl-2-hydroxypentan-triazolone group swings toward residues I45-I46 (Fig. 9B). Remarkably, points of posaconazole contact in the tunnel mouth are among mutation hot spots in azole resistant isolates of pathogenic fungi $A$. fumigatus [42,44-48] and C. albicans [43,49] (Fig. 8B and C).The scattered $\mathrm{F}_{\mathrm{o}}-\mathrm{F}_{\mathrm{c}}$ electron density map in the monomers $\mathrm{A}$ and $\mathrm{D}$ (Fig. 8A) suggests possible interconversion of the posaconazole conformers in dynamic equilibrium, meaning that the phenyl-2-hydroxypentan-triazolone group dangles in space within the tunnel mouth (Fig. 3D). Given the high sequence and structural similarities between $\mathrm{CYP} 51_{\mathrm{Tc}}$ and $\mathrm{CYP} 51_{\mathrm{Tb}}$, similar dynamics would be expected in the CYP5 $1_{\text {Tc }}$-posaconazole complex.

\section{Docking studies}

The x-ray structures of the CYP51 therapeutic targets determined in this work are intended for use in rational drug design. We also apply computational methods to explore binding modes of known chemical structures as well as to generate new scaffolds based on the configuration of the CYP51 binding sites. Considering the differential geometries of the host and pathogen binding sites, we aim to develop a pool of highly selective molecules with no cross-reactivity to human CYP51. As a first step, we docked posaconazole into the CYP51 $1_{\mathrm{Tc}}$ active site and compared the docking poses with the experimental structure of CYP51 ${ }_{\text {Tb-posaconazole complex. Two poses with similar docking }}$ scores were identified for posaconazole by GLIDE [39], differing primarily in the orientation of the 2,4-difluorophenyl ring (Fig. 10). Interestingly, the long posaconazole tail docks in a mode more similar to the CYP51 $1_{\mathrm{Tb}}$-posaconazole complex defined in this work rather than that in the recently deposited T. cruzi structure (PDB ID Code: 3K1O). Given that the protein-posaconazole interactions in the tunnel are of hydrophobic/aromatic stacking nature (Fig. 9), this ambiguity is not surprising.

Another source of docking ambiguity arises from the binding predicted for the 2,4-difluorophenyl substituent. In the better scoring pose 1 (highlighted yellow in Fig. 10), the 2,4difluorophenyl ring binds in the experimentally observed orientation 1 . In the slightly lower scoring pose 2 (highlighted pink), the 2,4-difluorophenyl ring is bound in a different pocket formed by the residues M106, E205, L208, F290, T295, L358 and M460, suggesting an additional cavity in the CYP51 active site suitable for drug targeting. This pose is achieved via flipping of the central furan ring to which all the substituents are attached. Thus, in addition to the experimentally observed binding ambiguity of the long substituent tail, conformational ambiguity of the difluorophenyl ring is predicted by the docking calculations and perhaps will be observed in future structures of CYP51 in complex with inhibitors similar to posaconazole.

\section{Implications for drug resistance}

The rapid development of azole resistance in T. cruzi observed in vitro suggests that the same may occur in patients [50]. Although no data are available on the development of posaconazole resistance in Chagas Disease patients, studies conducted on fungal infections indicate that posaconazole resistance occurs mainly by a mechanism involving mutation of the cyp51 gene $[42,51,52]$. Posaconazole appears to be less susceptible to the efflux pumps that confer resistance to some other azoles [43,51,53]. Mapping mutations in cyp51 genes in clinical posaconazole resistant isolates on the CYP51 structure, points to the tunnel entrance as a mutation hot spot. Mutations of G54, P216 and M220 in clinical 


\section{T.Cruzi_AAW47718}

T.cruzi_AAW4 7718

T.brucei_Q385E8

A. fumigatus_Q9P8R0

C. albicans P10613

Human_Q16850

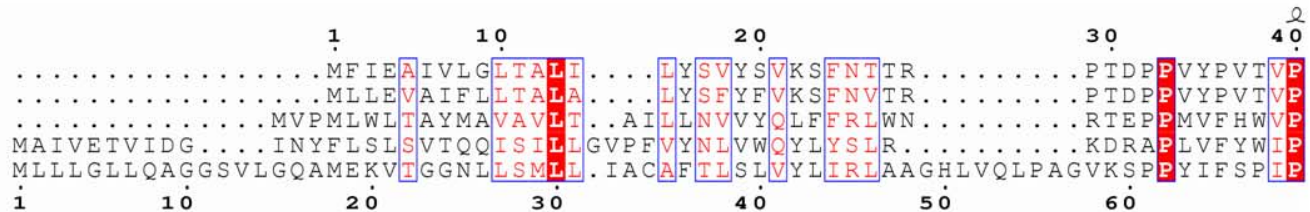

30
50
$6 \dot{0}$

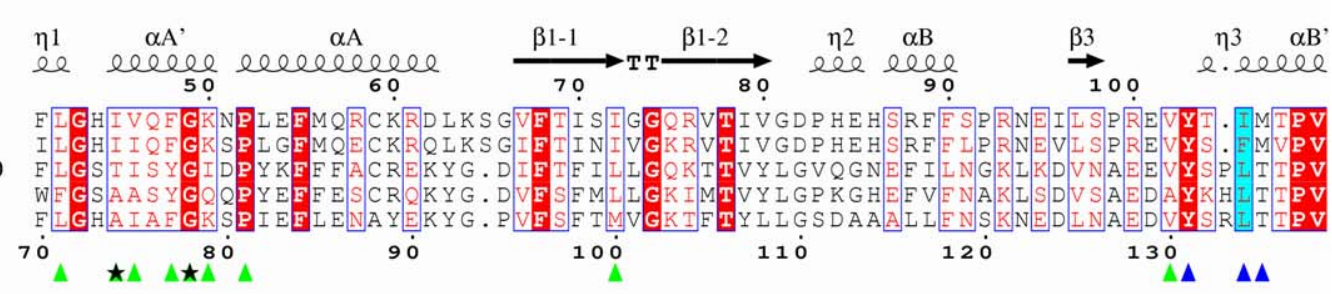

T.Cruzi_AAW47718

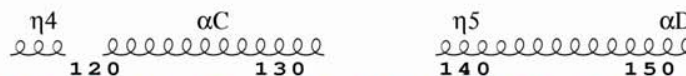

T.Cruzi_AAW47718 110

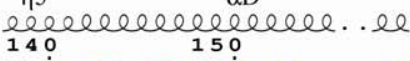

160

$\beta 2-1$

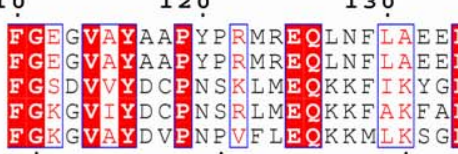

14

$\triangle 150^{\circ} \quad \triangle 160^{\circ}$

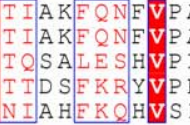

VPAIQHEVRKEMA. ENWK...EDEGVINLLEDCG AM

170

Human 016850

$\alpha \mathrm{E} \quad \alpha \mathrm{F}^{\prime} \quad \alpha \mathrm{F} \quad \alpha \mathrm{F}^{\prime \prime} \quad \eta 6 \quad \alpha \mathrm{C}$

T.Cruzi_AAW47718

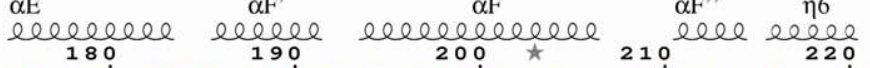

eleelee

lelelele

T.Cruzi_AAW47718

T.brucei 0385E 8

A. fumigatus Q9P8RO

C.albicans_P10613

Human_Q16850

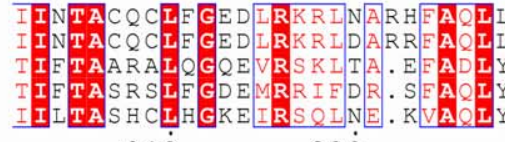

TIMES L I P A AVEMPW L L R L $230 \quad 240$

$\alpha \mathrm{H}$

$\alpha \mathrm{H}$

$\alpha \mathrm{I}$ $\eta 7$

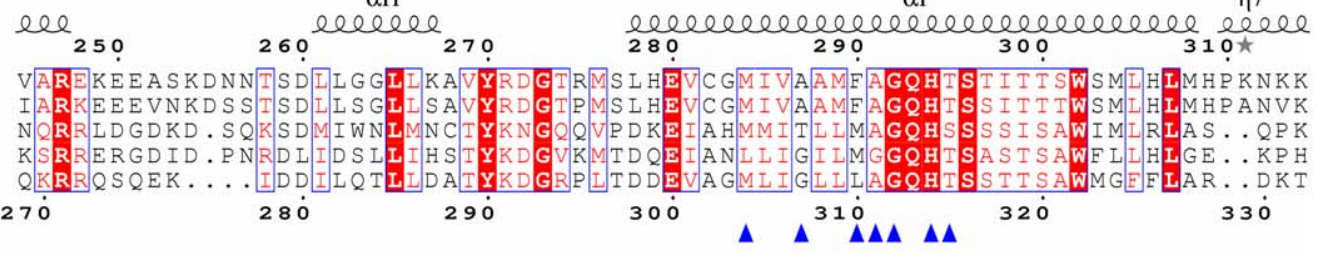

T.Cruzi_AAW4 7718

eloeleee

$\alpha \mathrm{K}^{\prime}$

ak

T.Cruzi_AAW4 7718

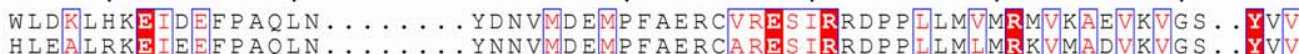
T.brucei $2385 \mathrm{E} 8$

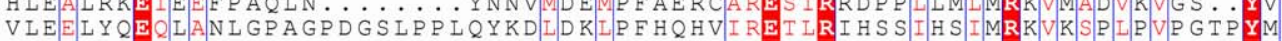

A. fumigatus 09P8R0

C.albicans P10613

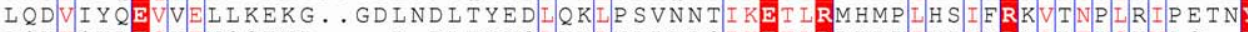

Human_Q16850

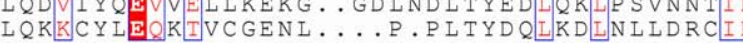

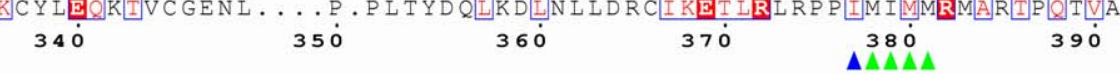

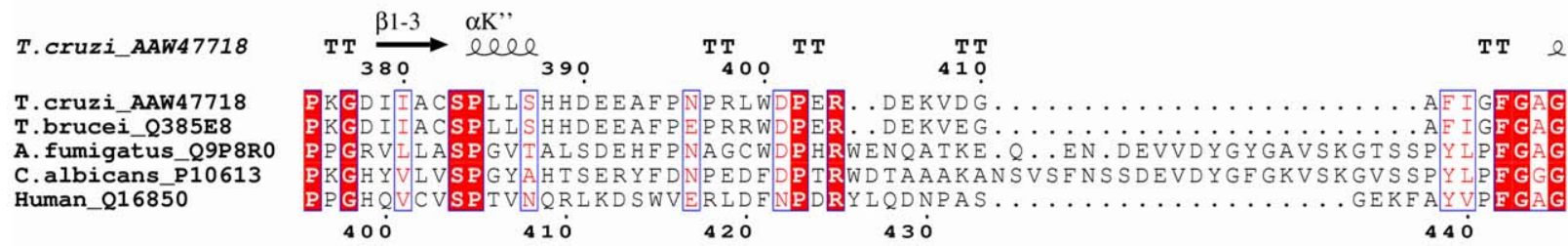

T.Cruzi_AAW47718

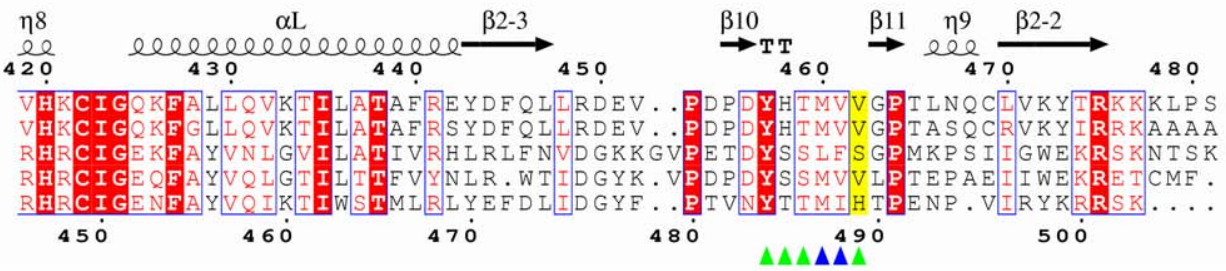

T.cruzi_AAW47718 T.brucei $0385 \mathrm{E} 8$

A. fumigatus Q9P8R C.albicans_P10613 Human_Q16850 
Figure 7. Sequence alignments between host and pathogen CYP51. Sequence alignments between CYP51 from Trypanosoma cruzi, Trypanosoma brucei, Aspergillus fumigatus, Candida albicans and human. Accession numbers of the proteins in the Swiss-Prot/TrEMBL (http://us. expasy.org/sprot) and NCBI (http://www.ncbi.nlm.nih.gov/) databases are given next to the name of the protein. Alignments were performed using CLUSTALW program online [61]. The figure was generated using ESPript [62]. The secondary structure annotation and residue numbering at the top correspond to CYP51 $1_{\mathrm{Tc}}$, residue numbering at the bottom corresponds to human CYP51. The $\alpha$-helices are labeled with capital letters according to generally accepted P450 nomenclature. The $\beta$-strands of large $\beta$-sheets are labeled with dashed numbers. Sequential numbers are used to label short two-residue $\beta$-strands. Residues within $7 \AA$ of fluconazole are labeled with blue triangles. Additional residues constituting the hydrophobic tunnel are labeled with green triangles. Human $\mathrm{H} 236$ and $\mathrm{H} 489$ and the corresponding residues in the pathogenic species are highlighted in yellow. Residues corresponding to CYP51 1 TC 1105 are highlighted in cyan. Mutation hot spots at the tunnel opening are marked with black stars. Gray stars highlight residues in alternate conformations.

doi:10.1371/journal.pntd.0000651.g007

isolates of $A$. fumigatus [42,44-48] (corresponding to G49, P2 10 and F214, respectively, in CYP5 $1_{\mathrm{Tc}}$ and $\mathrm{CYP} 51_{\mathrm{Tb}}$ ) and of A61 [49] and P230 [43] in clinical isolates of C. albicans (I45 and P210, respectively, in $\mathrm{CYP} 51_{\mathrm{Tc}}$ and $\mathrm{CYP} 51_{\mathrm{Tb}}$ ) map directly to the tunnel mouth (Fig. 8B and G). Mutations of G54 in A. fumigatus to arginine or tryptophan associate with moderate and high levels of resistance, respectively, and confer cross-resistance between itraconazole and posaconazole [44]. Mutations of M220 confer cross-resistance to all azole drugs tested, including itraconazole, voriconazole, ravuconazole and posaconazole [54,55] and therefore may interfere with the entry of the drugs. In accord with this assumption, posaconazole is reported to induce resistance to all

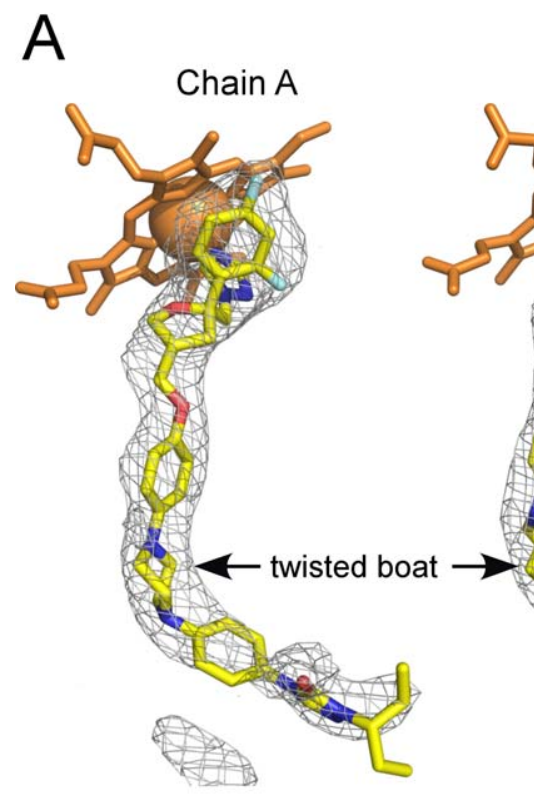

B

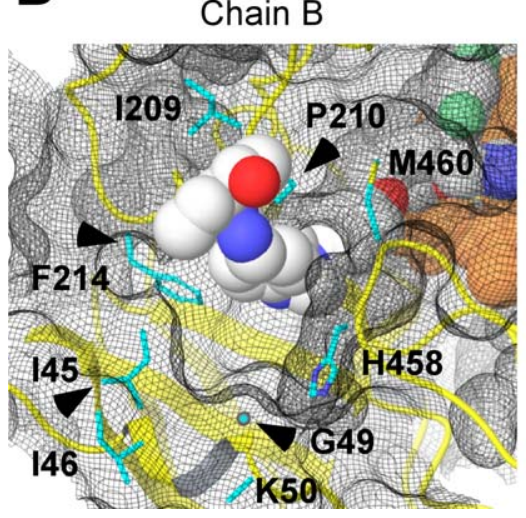

Chain B

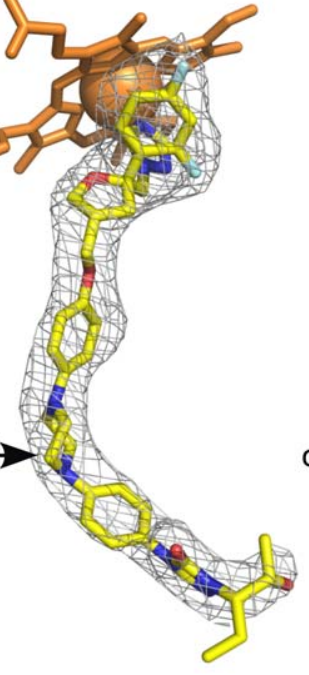

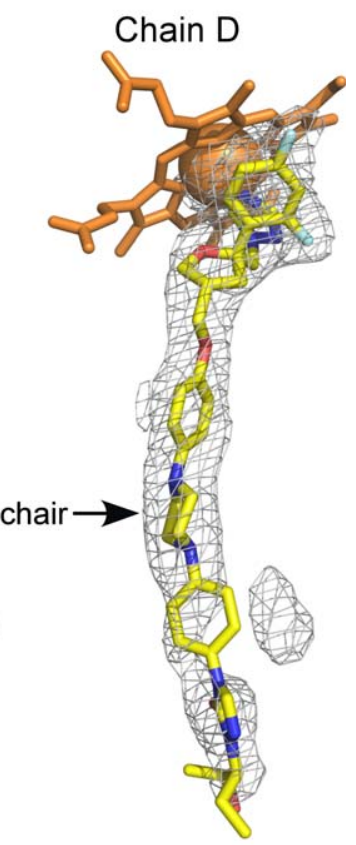

Chain D

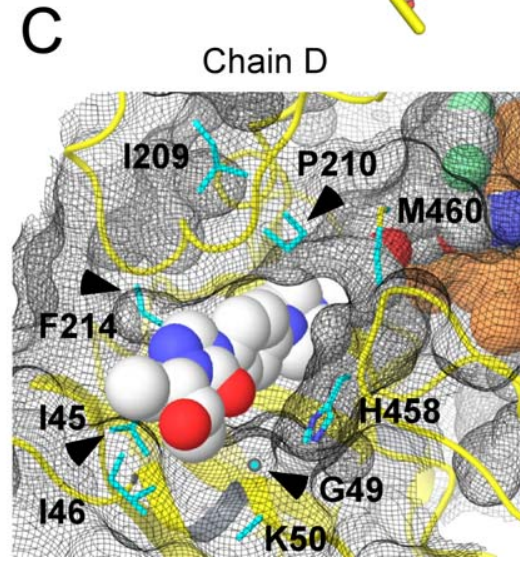

Figure 8. Posaconazole conformation. A. Fragments of the $F_{o}-F_{c}$ electron density map (grey mesh) in chains $A, B$ and $D$ calculated with the posaconazole coordinates omitted from the input file. B, C. Posaconazole protruding from the tunnel entrance is shown in the chain $B(B)$ and chain $\mathrm{D}(\mathbf{C})$. Protein backbone is depicted by yellow ribbon, protein surface is represented by black mesh, residues surrounding tunnel entrance (cyan) are in stick mode. Arrows point at the residues corresponding mutation hot spots in posaconazole resistant isolates of A. fumigatus and C. albicans. Posaconazole is shown by spheres with the carbon atoms white, oxygen red, nitrogen blue, fluorine light green. Heme in the background is orange. $\mathrm{K} 50$ side chain is not defined in the electron density and therefore modeled as alanine. Images were prepared using MAESTRO [63]. doi:10.1371/journal.pntd.0000651.g008 

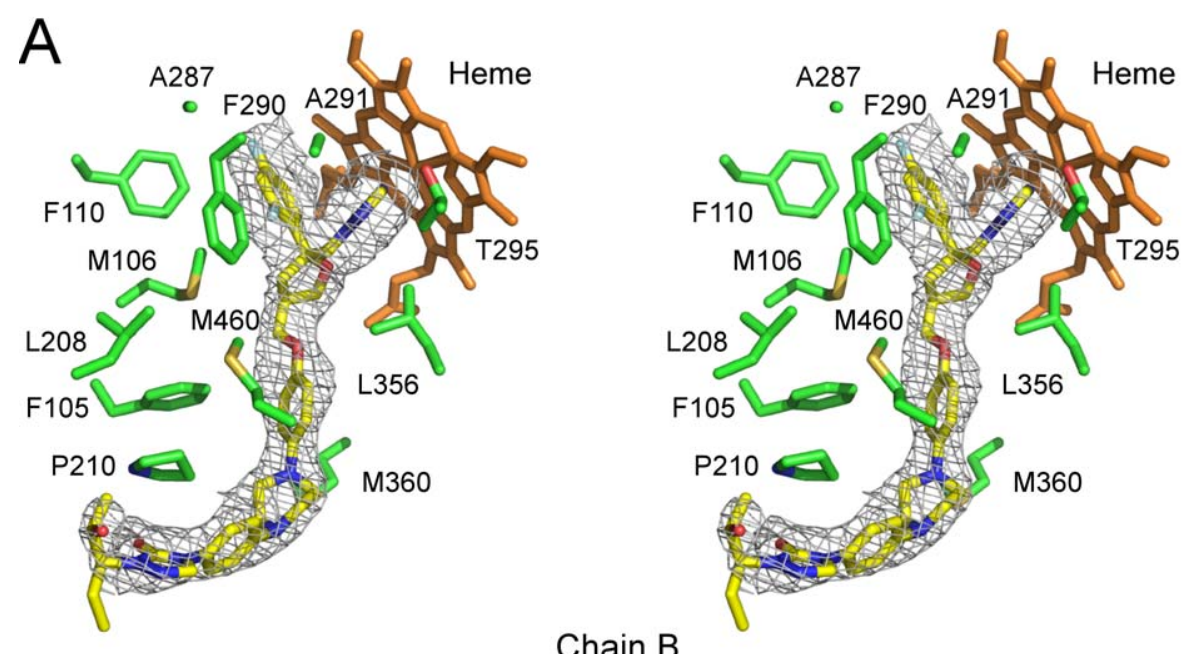

\section{Chain B}

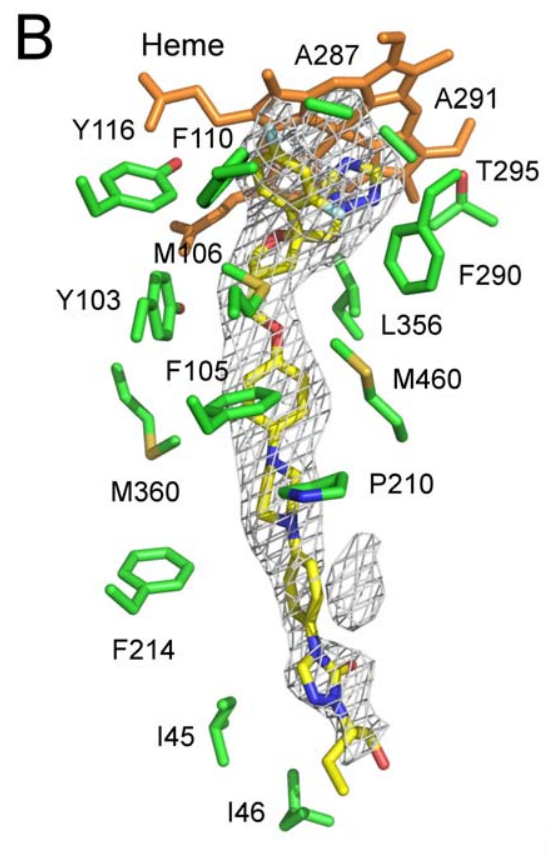

\section{Chain D}

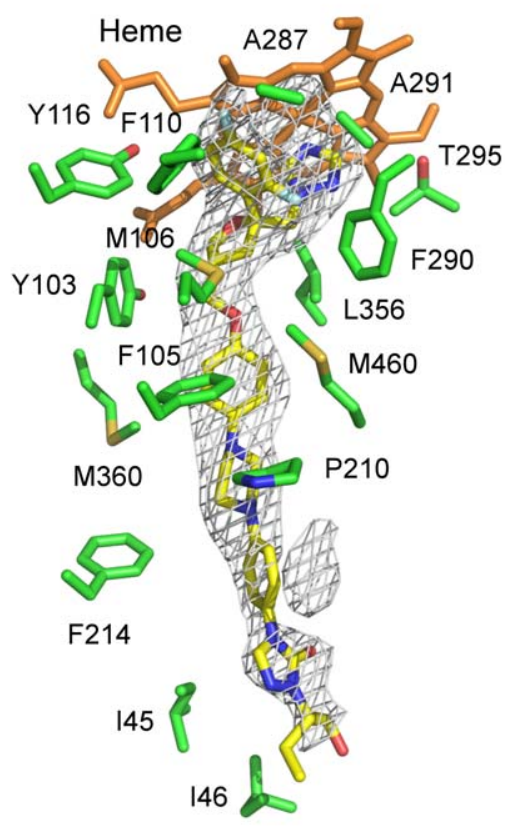

Figure 9. Posaconazole binding. Stereoscopic view of posaconazole in chain $B(\mathbf{A})$ and in chain $D(B)$ surrounded by the side chains within $4 \AA$. Image in B is rotated $\sim 90^{\circ}$ to the right along the vertical axis in the plane of drawing. To avoid image cluttering, four residues from this range, Y103, Y116, A211 and M360, were excluded from the view in A. Posaconazole carbons are highlighted in yellow, amino acid residues are in green. Otherwise color scheme is as in Fig. 8. doi:10.1371/journal.pntd.0000651.g009

azole drugs in Candida parapsilosis in vitro [51]. The alarming perspective emerging from antifungal therapy efforts must be taken into consideration when designing anti-Chagasic drugs targeting CYP5 $1_{\mathrm{Tc}}$. Thus, the terminal phenyl-2-hydroxypentantriazolone group in posaconazole may play an important role in pharmacokinetics rather than in the interactions with the target, and yet these interactions seem to induce resistance which otherwise could probably be avoided.

In summary, the x-ray structures of Trypanosoma CYP51 enzymes reported here open new opportunities for rationally designed inhibitors against therapeutic targets in important human pathogens. The structures provide templates for developing CYP51 inhibitors with improved efficacy and resistance properties that are structurally and synthetically simpler than posaconazole.
By utilizing the differential geometries between host and pathogen CYP51 binding sites, it maybe possible to create new drugs with minimized toxicity and host-pathogen cross-reactivity. In addition, the posaconazole binding mode offers insights into the development of drug resistance in pathogenic fungi, implying that an analogous mechanism may be implicated in protozoan pathogens. The reported structures also provide a good template for drug design targeting Leishmania CYP51. However, drug development must take into account the properties and accessibility of the compartment where these parasites reside. Unlike $T$. cruzi, Leishmania amastigotes replicate in the acidic environment $(\mathrm{pH}$ $\sim 5$ ) of the phagolysosomal vacuoles in macrophage cells [56,57], imposing different requirements on the physicochemical properties of CYP51 inhibitors targeting leishmaniasis. 

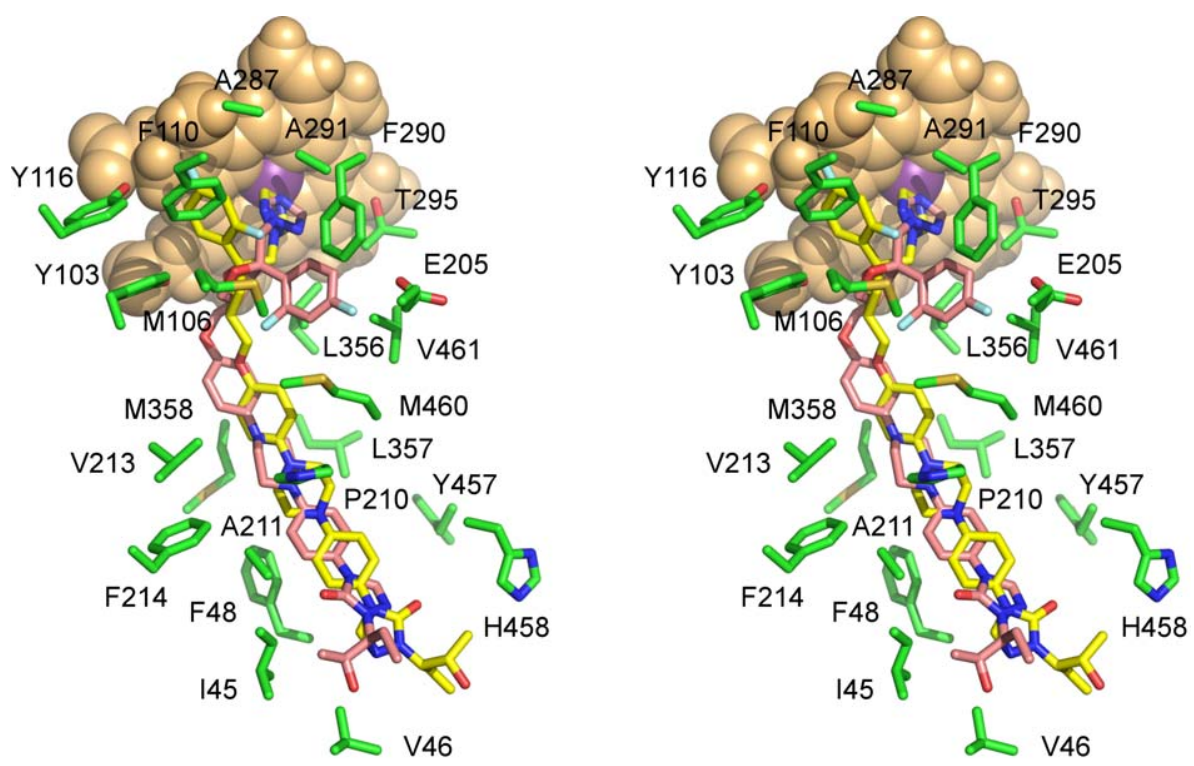

Figure 10. Posaconazole docking. Stereoscopic view of posaconazole docked in the active site CYP51 1 in pose 1 (yellow) and pose 2 (pink). Residues within $4 \AA$ of posaconazole (green) are shown. Binding ambiguity of the long posaconazole tail is likely due to the hydrophobic/aromatic stacking nature of the interactions in the substrate binding tunnel. Posaconazole and protein are shown by stick, heme by van der Waals spheres. Posaconazole carbon atoms are yellow or pink, oxygen red, nitrogen blue, sulfur yellow and fluorine pale cyan; heme is orange, For clarity, residues L208 and M360 are excluded from the view.

doi:10.1371/journal.pntd.0000651.g010

\section{Acknowledgments}

We thank Mr. Potter Wickware for critical reading of the manuscript, Prof. Paul Ortiz de Montellano and Dr. Clifford Bryant for valuable contributions, staff members of beamline 8.3.1 James Holton, George Meigs and Jane Tanamachi, the Advanced Light Source at Lawrence Berkeley National Laboratory for assistance with data collection.

\section{References}

1. Chagas C (1909) Nova trypanosomiase humana. Gaceta Medica da Bahia 40: 433-440.

2. World Health Organization (2002) Control of Chagas disease: second report of the WHO expert committee. Geneva: WHO.

3. Coura JR, De Castro SL (2002) A Critical Review on Chagas Disease Chemotherapy. Memórias do Instituto Oswaldo Cruz 97: 3-24.

4. McKerrow JH, Doyle PS, Engel JC, Podust LM, Robertson SA, et al. (2009) Two approaches to discovering and developing new drugs for Chagas disease. Mem Inst Oswaldo Cruz 104: 263-269.

5. Roberts CW, McLeod R, Rice DW, Ginger M, Chance ML, et al. (2003) Fatty acid and sterol metabolism: potential antimicrobial targets in apicomplexan and trypanosomatid parasitic protozoa. Mol Biochem Parasitol 126: 129-142.

6. Urbina JA, Docampo R (2003) Specific chemotherapy of Chagas disease: controversies and advances. Trends Parasitol 19: 495-501.

7. Buckner F (2008) Sterol 14-demethylase inhibitors for Trypanosoma cruzi infections. In: Majumder HK, ed. Drug Targets in Kinetoplastid Parasites. New York: Springer. pp 61-80.

8. de Souza W, Rodrigues JC (2009) Sterol Biosynthesis Pathway as Target for Anti-trypanosomatid Drugs. Interdiscip Perspect Infect Dis 2009. 642502 p.

9. Kauffman CA, Malani AN, Easley C, Kirkpatrick P (2007) Posaconazole. Nat Rev Drug Discov 6: 183-184

10. Molina J, Martins-Filho O, Brener Z, Romanha AJ, Loebenberg D, et al. (2000) Activities of the triazole derivative SCH 56592 (posaconazole) against drugresistant strains of the protozoan parasite Trypanosoma (Schizotrypanum) cruzi in immunocompetent and immunosuppressed murine hosts. Antimicrob Agents Chemother 44: 150-155.

11. Ferraz ML, Gazzinelli RT, Alves RO, Urbina JA, Romanha AJ (2007) The antiTrypanosoma cruzi activity of posaconazole in a murine model of acute Chagas' disease is less dependent on gamma interferon than that of benznidazole. Antimicrob Agents Chemother 51: 1359-1364.

12. Morris MI (2009) Posaconazole: a new oral antifungal agent with an expanded spectrum of activity. Am J Health Syst Pharm 66: 225-236.

13. Buckner F, Yokoyama K, Lockman J, Aikenhead K, Ohkanda J, et al. (2003) A class of sterol 14-demethylase inhibitors as anti-Trypanosoma cruzi agents. Proc Natl Acad Sci U S A 100: 15149-15153.

\section{Author Contributions}

Conceived and designed the experiments: MPJ LMP. Performed the experiments: CKG SSFL LMP. Analyzed the data: CKG SSFL GG LMP. Contributed reagents/materials/analysis tools: CG MPJ JHM LMP. Wrote the paper: JHM LMP.

14. Hucke O, Gelb MH, Verlinde CL, Buckner FS (2005) The protein farnesyltransferase inhibitor Tipifarnib as a new lead for the development of drugs against Chagas disease. J Med Chem 48: 5415-5418.

15. Suryadevara PK, Olepu S, Lockman JW, Ohkanda J, Karimi M, et al. (2009) Structurally simple inhibitors of lanosterol 14alpha-demethylase are efficacious in a rodent model of acute Chagas disease. J Med Chem 52: 3703-3715.

16. Chen C-K, Doyle PS, Yermalitskaya LV, Mackey ZB, Ang KKH, et al. (2009) Trypanosoma cruzi CYP51 inhibitor derived from a Mycobacterium tuberculosis screen hit. PLoS Negl Trop Dis 3: e372.

17. Chennamaneni NK, Arif J, Buckner FS, Gelb MH (2009) Isoquinoline-based analogs of the cancer drug clinical candidate tipifarnib as anti-Trypanosoma cruzi agents. Bioorg Med Chem Lett 19: 6582-6584.

18. Podust LM, Poulos TL, Waterman MR (2001) Crystal structure of cytochrome P450 14alpha-sterol demethylase (CYP51) from Mycobacterium tuberculosis in complex with azole inhibitors. Proc Natl Acad Sci USA 98: 3068-3073.

19. Podust LM, Yermalitskaya LV, Lepesheva GI, Podust VN, Dalmasso EA, et al. (2004) Estriol bound and ligand-free structures of sterol 14alpha-demethylase. Structure 12: 1937-1945.

20. Nasser Eddine A, von Kries JP, Podust MV, Warrier T, Kaufmann SH, et al. (2008) X-ray structure of $4,4^{\prime}$-dihydroxybenzophenone mimicking sterol substrate in the active site of sterol 14alpha-demethylase (CYP51). J Biol Chem 283: $15152-15159$

21. Lepesheva GI, Zaitseva NG, Nes WD, Zhou W, Arase M, et al. (2006) CYP51 from Trypanosoma cruzi: a phyla-specific residue in the B' helix defines substrate preferences of sterol 14alpha-demethylase. J Biol Chem 281: 3577-3585.

22. Marichal P, Koymans L, Willemsens S, Bellens D, Verhasselt P, et al. (1999) Contribution of mutations in the cytochrome P450 14alpha-demethylase (Erg11p, Cyp51p) to azole resistance in Candida albicans. Microbiology 145: 2701-2713.

23. Sanglard D, Ischer F, Koymans L, Bille J (1998) Amino acid substitutions in the cytochrome P450 lanosterol 14alpha-demethylase (CYP51) from azole- resistant Candida albicans clinical isolates contributing to the resistance to azole antifungal agents. Antimicrob Agents Chemother 42: 241-253.

24. Asai K, Tsuchimori N, Okonogi K, Perfect JR, Gotoh O, et al. (1999) Formation of azole-resistant Candida albicans by mutation of sterol 14-demethylase P450. Antimicrob Agents Chemother 43: 1163-1169. 
25. Kelly SL, Lamb DC, Kelly DE (1999) Y132H substitution in Candida albicans sterol 14alpha-demethylase confers fluconazole resistance by preventing binding to haem. FEMS Microbiol Lett 180: 171-175.

26. Perea S, Lopez-Ribot JL, Kirkpatrick WR, McAtee RK, Santillan RA, et al. (2001) Prevalence of molecular mechanisms of resistance to azole antifungal agents in Candida albicans strains displaying high-level fluconazole resistance isolated from human immunodeficiency virus-infected patients. Antimicrob Agents Chemother 45: 2676-2684.

27. Kudo M, Ohi M, Aoyama Y, Nitahara Y, Chung SK, et al. (2005) Effects of $\mathrm{Y} 132 \mathrm{H}$ and $\mathrm{F} 145 \mathrm{~L}$ substitutions on the activity, azole resistance and spectral properties of Candida albicans sterol 14-demethylase P450 (CYP51): a live example showing the selection of altered P450 through interaction with environmental compounds. J Biochem (Tokyo) 137: 625-632.

28. Wheat LJ, Connolly P, Smedema M, Durkin M, Brizendine E, et al. (2006) Activity of newer triazoles against Histoplasma capsulatum from patients with AIDS who failed fluconazole. J Antimicrob Chemother 57: 1235-1239.

29. Chau AS, Chen G, McNicholas PM, Mann PA (2006) Molecular basis for enhanced activity of posaconazole against Absidia corymbifera and Rhizopus oryzae. Antimicrob Agents Chemother 50: 3917-3919.

30. El-Sayed NM, Myler PJ, Blandin G, Berriman M, Crabtree J, et al. (2005) Comparative genomics of trypanosomatid parasitic protozoa. Science 309: $404-409$.

31. Coppens I, Courtoy PJ (2000) The adaptative mechanisms of Trypanosoma brucei for sterol homeostasis in its different life-cycle environments. Annu Rev Microbiol 54: 129-156.

32. Barnes HJ, Arlotto MP, Waterman MR (1991) Expression and enzymatic activity of recombinant cytochrome P450 17 alpha-hydroxylase in Escherichia coli. Proc Natl Acad Sci U S A 88: 5597-5601.

33. Omura T, Sato R (1964) The carbon monoxide-binding pigment of liver microsomes. II. Solubilization, purification, and properties. J Biol Chem 239: 2379-2385.

34. Holton J, Alber T (2004) Automated protein crystal structure determination using ELVES. Proc Natl Acad Sci U S A 101: 1537-1542.

35. Cowtan K (2006) The Buccaneer software for automated model building. 1 . Tracing protein chains. Acta Crystallogr D Biol Crystallogr 62: 1002-1011.

36. Emsley P, Cowtan K (2004) Coot: model-building tools for molecular graphics. Acta Crystallogr D Biol Crystallogr 60: 2126-2132.

37. Murshudov GN, Vagin AA, Dodson EJ (1997) Refinement of macromolecular structures by the maximum-likelihood method. Acta Crystallogr D Biol Crystallogr 53: 240-255.

38. Winn MD, Isupov MN, Murshudov GN (2001) Use of TLS parameters to model anisotropic displacements in macromolecular refinement. Acta Crystallogr D Biol Crystallogr 57: 122-133.

39. Friesner RA, Banks JL, Murphy RB, Halgren TA, Klicic JJ, et al. (2004) Glide: A new approach for rapid, accurate docking and scoring. 1. Method and assessment of docking accuracy. J Med Chem 47: 1739-1749.

40. Zhu K, Shirts MR, Friesner RA, Jacobson MP (2007) Multiscale optimization of a truncated Newton minimization algorithm and application to proteins and protein-ligand complexes. J Chem Theory Comput 3: 640-648.

41. Podust LM, Stojan J, Poulos TL, Waterman MR (2001) Substrate recognition sites in 14alpha-sterol demethylase from comparative analysis of amino acid sequences and X-ray structure of Mycobacterium tuberculosis CYP51. J Inorg Biochem 87: 227-235.

42. Howard SJ, Cerar D, Anderson MJ, Albarrag A, Fisher MC, et al. (2009) Frequency and evolution of azole resistance in Aspergillus fumigatus associated with treatment failure. Emerg Infect Dis 15: 1068-1076.

43. Li X, Brown N, Chau AS, Lopez-Ribot JL, Ruesga MT, et al. (2004) Changes in susceptibility to posaconazole in clinical isolates of Candida albicans. J Antimicrob Chemother 53: 74-80.

44. Mann PA, Parmegiani RM, Wei SQ, Mendrick CA, Li X, et al. (2003) Mutations in Aspergillus fumigatus resulting in reduced susceptibility to posacona- zole appear to be restricted to a single amino acid in the cytochrome P450 14alpha-demethylase. Antimicrob Agents Chemother 47: 577-581.

45. Diaz-Guerra TM, Mellado E, Cuenca-Estrella M, Rodriguez-Tudela JL (2003) A point mutation in the 14alpha-sterol demethylase gene cyp51A contributes to itraconazole resistance in Aspergillus fumigatus. Antimicrob Agents Chemother 47: $1120-1124$.

46. Nascimento AM, Goldman GH, Park S, Marras SA, Delmas G, et al. (2003) Multiple resistance mechanisms among Aspergillus fumigatus mutants with highlevel resistance to itraconazole. Antimicrob Agents Chemother 47: 1719-1726.

47. da Silva Ferreira ME, Capellaro JL, dos Reis Marques E, Malavazi I, Perlin D, et al. (2004) In vitro evolution of itraconazole resistance in Aspergillus fumigatus involves multiple mechanisms of resistance. Antimicrob Agents Chemother 48: 4405-4413.

48. Chen J, Li H, Li R, Bu D, Wan Z (2005) Mutations in the cyp51A gene and susceptibility to itraconazole in Aspergillus fumigatus serially isolated from a patient with lung aspergilloma. J Antimicrob Chemother 55: 31-37.

49. Xiao L, Madison V, Chau AS, Loebenberg D, Palermo RE, et al. (2004) Threedimensional models of wild-type and mutated forms of cytochrome P450 14alpha-sterol demethylases from Aspergillus fumigatus and Candida albicans provide insights into posaconazole binding. Antimicrob Agents Chemother 48: 568-574.

50. Buckner FS, Wilson AJ, White TC, Van Voorhis WC (1998) Induction of resistance to azole drugs in Trypanosoma cruzi. Antimicrob Agents Chemother 42: 3245-3250.

51. Pinto e Silva AT, Costa-de-Oliveira S, Silva-Dias A, Pina-Vaz C, Rodrigues AG (2009) Dynamics of in vitro acquisition of resistance by Candida parapsilosis to different azoles. FEMS Yeast Res 9: 626-633.

52. Pfaller MA, Diekema DJ, Ghannoum MA, Rex JH, Alexander BD, et al. (2009) Wild-type MIC distribution and epidemiological cutoff values for Aspergillus fumigatus and three triazoles as determined by the Clinical and Laboratory Standards Institute broth microdilution methods. J Clin Microbiol 47: 3142-3146.

53. Chau AS, Mendrick CA, Sabatelli FJ, Loebenberg D, McNicholas PM (2004) Application of real-time quantitative PCR to molecular analysis of Candida albicans strains exhibiting reduced susceptibility to azoles. Antimicrob Agents Chemother 48: 2124-2131.

54. Mellado E, Garcia-Effron G, Alcazar-Fuoli L, Cuenca-Estrella M, RodriguezTudela JL (2004) Substitutions at methionine 220 in the 14alpha-sterol demethylase (Cyp51A) of Aspergillus fumigatus are responsible for resistance in vitro to azole antifungal drugs. Antimicrob Agents Chemother 48: 2747-2750.

55. Rodriguez-Tudela JL, Alcazar-Fuoli L, Mellado E, Alastruey-Izquierdo A, Monzon A, et al. (2008) Epidemiological cutoffs and cross-resistance to azole drugs in Aspergillus fumigatus. Antimicrob Agents Chemother 52: 2468-2472.

56. Antoine JC, Prina E, Jouanne C, Bongrand P (1990) Parasitophorous vacuoles of Leishmania amazonensis-infected macrophages maintain an acidic $\mathrm{pH}$. Infect Immun 58: 779-787.

57. Antoine J-C, Prina E, Lang T, Courret N (1998) The biogenesis and properties of the parasitophorous vacuoles that harbour Leishmania in murine macrophages. Trends in Microbiology 6: 392-401.

58. DeLano WL (2002) The PyMOL molecular graphics system. San Carlos, CA, USA: DeLano Scientific

59. Humphrey W, Dalke A, Schulten K (1996) VMD: visual molecular dynamics. J Mol Graph 14: 33-38.

60. Pettersen EF, Goddard TD, Huang CC, Couch GS, Greenblatt DM, et al. (2004) UCSF Chimera-a visualization system for exploratory research and analysis. J Comput Chem 25: 1605-1612.

61. Thompson JD, Higgins DG, Gibson TJ (1994) CLUSTAL W: improving the sensitivity of progressive multiple sequence alignment through sequence weighting, position-specific gap penalties and weight matrix choice. Nucleic Acids Res 22: 4673-4680.

62. Gouet P, Courcelle E, Stuart DI, Metoz F (1999) ESPript: multiple sequence alionments in PostScript. Bioinformatics 15: 305-308.

63. Maestro. New York, NY, USA: Schrodinger, LLC. 